\title{
Three-dimensional coherence of light speckles: Experiment
}

\author{
D. Magatti, A. Gatti, and F. Ferri* \\ Dipartimento di Fisica e Matematica and CNR-INFM-CNISM, Universita' dell'Insubria, via Valleggio 11, 22100 Como, Italy
}

(Received 11 March 2009; published 18 May 2009)

\begin{abstract}
We provide an experimental detailed study of the three-dimensional coherence properties of light speckles produced by different tunable pseudothermal sources. Our findings confirm the theoretical prediction of the companion article [A. Gatti et al., Phys. Rev. A 78, 063806 (2008)], according to which the longitudinal coherence of the speckles is ruled by ordinary diffraction laws only in the deep-Fresnel zone close to the source, deviates from this behavior in the Fresnel zone, and tends to become infinite when approaching the Fraunhofer zone. A quantitative comparison with theory is presented for Gaussian speckles in all the three regimes and for Airy speckles in the deep-Fresnel zone. Potential applications to three-dimensional imaging techniques are briefly discussed.
\end{abstract}

DOI: 10.1103/PhysRevA.79.053831

PACS number(s): 42.25.Kb, 42.30.Ms

\section{INTRODUCTION}

The three-dimensional (3D) spatial coherence properties of light speckles have been the subject of a theoretical article appeared on this journal a few months ago [1]. In that paper we provided a detailed description of the problem, working out the expression for the 3D degree of mutual coherence $\mu$ for any distance $z$ from the source, encompassing the deepFresnel zone close to the source, the Fresnel zone, up to the Fraunhofer zone. Our work started from what was already known about the two-dimensional (2D) properties of the transversal coherence (thoroughly described in the literature, see [2-4], and references therein) and generalized these results to the 3D case, mainly focusing onto the longitudinal properties of $\mu$, which, conversely, have been the subject of only few investigations in the literature [5-7]. The full 3D picture emerging from literature results and from our findings shows that the behavior of $\mu$ is quite different in these three regimes:

(i) In the deep-Fresnel zone the transversal coherence proprieties of the speckles are invariant upon propagation and mirror the coherence properties of the source. These speckles (called also "near field" speckles, after Giglio et al., who first observed them [8-10]) have a transversal size equal to the size of the "grains" belonging to the source and, if the source is planar (i.e., characterized by a flat average phase profile), retain the same size upon propagation. In Ref. [1] we demonstrated that also the longitudinal coherence properties of $\mu$ are invariant upon distance and they are governed by an ordinary diffractive behavior, leading to the result that the speckle length scales as $\delta z \sim \delta x^{2} / \lambda, \delta x$ being the transverse speckle size.

(ii) In the Fresnel zone the speckles become progressively larger and longer. Their transversal coherence properties are ruled by the well known generalized Van Cittert-Zernike (VCZ) theorem [11,12], while their longitudinal properties appear to be still governed by the ordinary diffractive behavior. Thus, as soon as $\delta x>\lambda$, the speckles are much longer than wider, and for this reason they are sometimes pictured

\footnotetext{
*Corresponding author; fabio.ferri@uninsubria.it
}

in the literature [5-7] as "jelly-bean-like" coherence volumes. However, we demonstrated in Ref. [1] that the latter behavior is not completely correct because it is obtained under the assumption that the speckle length is much smaller than the distance from the source. When this distance becomes comparable with the Fraunhofer distance, the above assumption fails and the speckles become progressively elongated, with their longitudinal size longer than expected from pure diffraction. We pointed out that this behavior is due to the overall curvature of the average speckle beam, which makes the speckle separate angularly at a rate comparable with the rate at which they overlap because of diffraction.

(iii) In the Fraunhofer zone, the speckles are (transversally) so large that their diffraction angle becomes negligible with respect to the angle at which they separate because of the beam curvature. Thus, the speckles spread apart at the same rate at which they broaden, they never overlap, and tend to become truly infinite in length.

The aim of this companion paper is to carry out a detailed experimental characterization of the 3D-coherence volume of the light speckles in the three regimes mentioned above. The problem of studying the speckles in such disparate regimes will be tackled by employing different pseudothermal sources whose coherence properties can be easily tuned, with the opportunity of producing speckles with the desired characteristics. In all the three regimes, we shall compare quantitatively our findings with the theoretical predictions reported in Ref. [1] for the case of Gaussian speckles. In particular, while the purely diffractive behavior of jelly-beanlike speckles has been the subject of previous experimental investigations $[5,6]$, our prediction concerning the deviation from this behavior on approaching the Fraunhofer zone has never been tested before. In this work we shall demonstrate a remarkable deviation from the purely diffractive behavior at distances from the source accessible to experimental implementations. In addition we shall also realize and characterize "Airy" speckles, i.e., speckles whose coherence factor is equal to the Airy function. When produced in the deepFresnel zone, these speckles show a highly complex 3Dcoherence structure, which can be described in terms of the point spread function (PSF) of a classical wide angle optical microscope. 
This paper is organized as follows: in Sec. II we recall the main definitions used in coherence optics for characterizing the properties of a speckle field and describe the features of the planar thermal sources used in the experiment; Sec. III summarizes the main theoretical results presented and discussed in Ref. [1]; Sec. IV is devoted to Gaussian speckles in the deep-Fresnel zone and compares our experimental results with the analytical expression in Ref. [1]; Sec. V carries out the same analysis for Gaussian speckles in the VCZ regime, investigating both the Fresnel and the Fraunhofer zones; Sec. VI is devoted to the study of Airy speckles in the deepFresnel zone and shows the remarkable difference between the 3D morphology of these speckles and Gaussian speckles. Conclusions, potential applications, and perspectives are presented in Sec. VII.

\section{THEORETICAL BACKGROUND AND SOURCE PROPERTIES}

In this section we first (Sec. II A) recall the main definitions and assumptions commonly used in coherence optics for characterizing the coherence/statistical properties of a speckle field and then (Sec. II B) we report the main features of the source generating such a field.

\section{A. Theoretical background}

The 3D-coherence properties of the speckle field emitted by a planar thermal source, located at the plane $z=0$, can be characterized in terms of the mutual coherence function [3],

$$
G^{(1)}\left(\mathbf{x}_{1}, z_{1} ; \mathbf{x}_{2}, z_{2}\right) \equiv\left\langle A^{*}\left(\mathbf{x}_{1}, z_{1}\right) A\left(\mathbf{x}_{2}, z_{2}\right)\right\rangle,
$$

in which $A\left(\mathbf{x}_{1}, z_{1}\right)$ and $A\left(\mathbf{x}_{2}, z_{2}\right)$ are the electric fields at the two $3 \mathrm{D}$ points $\left(\mathbf{x}_{1}, z_{1}\right)$ and $\left(\mathbf{x}_{2}, z_{2}\right)$. Here $\mathbf{x}$ represents the transverse $2 \mathrm{D}$ coordinate while $z$ is the distance from the source and $\langle\cdots\rangle$ means a statistical average. Assuming that $A(\mathbf{x}, z)$ is characterized by a circular Gaussian statistics, a Siegert-type relation holds [3],

$$
\left\langle I\left(\mathbf{x}_{1}, z_{1}\right) I\left(\mathbf{x}_{2}, z_{2}\right)\right\rangle=\left\langle I\left(\mathbf{x}_{1}, z_{1}\right)\right\rangle\left\langle I\left(\mathbf{x}_{2}, z_{2}\right)\right\rangle\left[1+\left|\mu\left(\mathbf{x}_{1}, z_{1} ; \mathbf{x}_{2}, z_{2}\right)\right|^{2}\right],
$$

in which

$$
\mu\left(\mathbf{x}_{1}, z_{1} ; \mathbf{x}_{2}, z_{2}\right)=\frac{G^{(1)}\left(\mathbf{x}_{1}, z_{1} ; \mathbf{x}_{2}, z_{2}\right)}{\sqrt{\left\langle I\left(\mathbf{x}_{1}, z_{1}\right)\right\rangle\left\langle I\left(\mathbf{x}_{2}, z_{2}\right)\right\rangle}}
$$

is a dimensionless quantity called degree of mutual coherence or sometimes complex coherence factor [13] and $\langle I(\mathbf{x}, z)\rangle=G^{(1)}(\mathbf{x}, z ; \mathbf{x}, z)$ is the average intensity at the point $(\mathbf{x}, z)$. The function $\mu$ is normalized to one $\left[|\mu(\mathbf{x}, z ; \mathbf{x}, z)|^{2}\right.$ $=1]$ and its squared modulus $|\mu|^{2}$ can be easily measured in an experiment.

\section{B. Source properties}

In Ref. [1] we assumed to have a so-called planar quasihomogeneous source, that is, a planar source characterized by two distinct length scales: a small length scale $\delta x_{0}$ related to the size of the transverse spatial correlations at the source plane and a large length scale $D$ related to overall transverse size of the source. When $\delta x_{0} \ll D$, the mutual coherence function of the source located at the plane $z=0$ can be factorized as

$$
G^{(1)}\left(\mathbf{x}_{1}, 0 ; \mathbf{x}_{2}, 0\right)=I_{0}\left(\frac{\mathbf{x}_{1}+\mathbf{x}_{2}}{2}\right) C_{0}\left(\mathbf{x}_{2}-\mathbf{x}_{1}\right)
$$

in which $I_{0}\left[\left(\mathbf{x}_{1}+\mathbf{x}_{2}\right) / 2\right]$ is the large average intensity profile of width $\sim D$ emitted by the source, which modulates the narrow $C_{0}\left(\mathbf{x}_{2}-\mathbf{x}_{1}\right)$ correlation function of width $\sim \delta x_{0}$, characterizing the source inhomogeneities. $C_{0}$ is normalized to unity $\left[C_{0}(0)=1\right]$ and is a real function, meaning that the average phase profile of the field at the source plane is flat.

Experimentally, a planar quasihomogeneous source can be realized by focusing a Gaussian laser beam onto a thin scattering medium, such as a plane diffuser or a ground glass. Typically a diffuser is characterized by surface "grains" (the size of its roughness) on the order of a few microns, and therefore the focused beam should have a waist $D$ $\sim 100 \mu \mathrm{m}$ or larger. This method is quite simple but exhibits a strong limitation due to the fact that, while the size $D$ of the source can be easily tuned, the size $\delta x_{0}$ of the speckles at the source plane is fixed by the microscopic details of the particular diffuser being used. A much more flexible method can be arranged by collecting the light scattered by a diffuser with a Fourier lens placed at a distance equal to its focal length. Thus, in the focal plane of the lens the coherence factor of the speckles is given by the Fourier transform of the intensity distribution illuminating the diffuser. In this way, we can form speckles with the desired shape (for example, Gaussian or Airy speckles, see Secs. IV and VI) and tunable size $\delta x_{0}$, the latter one being controlled by the size $w_{0}$ of the beam impinging onto the diffuser. The focal plane of the lens becomes, therefore, the $z=0$ plane of a synthetic thermal source, whose granular structure is set by the speckle size $\delta x_{0}$ and whose transverse size can be easily limited by inserting a diaphragm of diameter $D$. The average phase profile across the $z=0$ plane is controlled by the curvature of the beam impinging onto the diffuser. Thus, if the beam is collimated, the corresponding phase profile of the speckles is flat and produces a planar source, as required for generating deep-Fresnel speckles. A detailed description of this kind of "Fourier transform" thermal sources will be given in Secs. IV and VI.

\section{MAIN RESULTS WORKED OUT IN REF. [1]}

The overall 3D spatial coherence properties of light speckles are synthetically illustrated in Fig. 7 of Ref. [1]. This figure summarizes what was already know about the transversal properties of the speckles [2-11] and reports our results relative to the longitudinal properties. All the 3D properties can be summarized as follows:

(1) As to the transverse coherence properties of the speckle field, the half plane $z>0$ can be separated into two zones, divided by the crossover distance, 


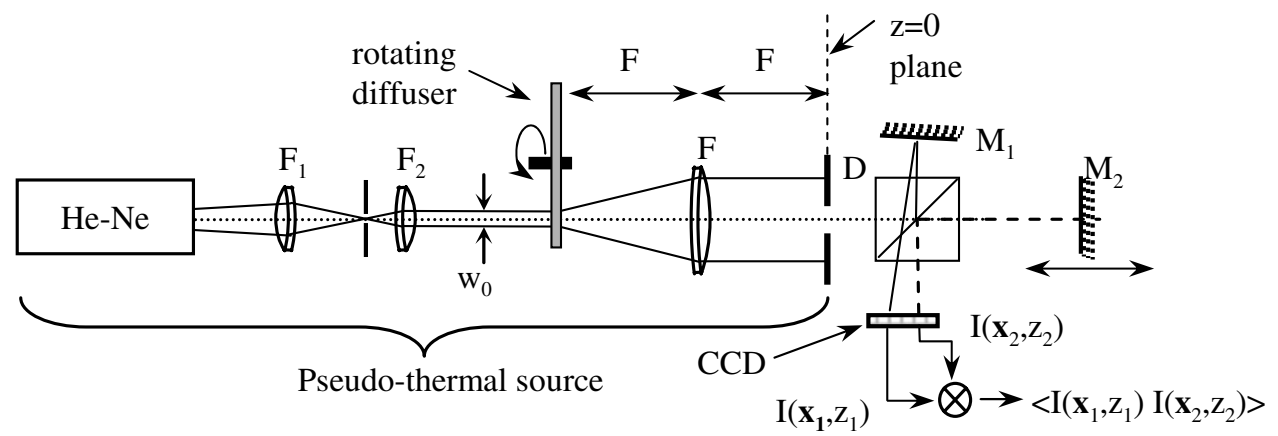

FIG. 1. Schematic diagram of the setup used for studying 3D Gaussian speckles in the deep-Fresnel zone.

$$
z_{\mathrm{VCZ}}=D \delta x_{0} / \lambda
$$

where $D$ is the transverse size of the source and $\delta x_{0}$ is the speckle size at the source plane (see Sec. II B). In these two zones quite different behaviors are expected:

(i) Close to the source $\left(z \ll z_{\mathrm{VCZ}}\right)$ we are in the deepFresnel zone, where the transversal coherence proprieties of the speckles are invariant upon propagation and mirror the coherence properties of the source $[1,10]$. In particular the transverse size of the speckles is the same as the one at the source plane $^{1}$ (Eq. 15 of Ref. [1]),

$$
\delta x_{n}=\delta x_{0} .
$$

(ii) Far from the source $\left(z \gg z_{\mathrm{VCZ}}\right)$ we are in the Van Cittert-Zernike zone, where the transverse coherence is ruled by the (generalized) Van Cittert-Zernike theorem [11,12], according to which the transverse speckle size increases linearly with the distance from the source as

$$
\delta x_{f} \sim \frac{\lambda z}{D} .
$$

As predicted by the VCZ theorem, the speckles size does not carry any information on the microscopic source properties but depends only on its transversal size $D$.

(2) As to the longitudinal coherence properties, the half plane $z>0$ can be divided in three zones, delimited by $z_{\mathrm{VCZ}}$ and by the farthest away distance,

$$
z_{\mathrm{Fr}}=D^{2} / \lambda
$$

which corresponds to the Fraunhofer distance of the source and fall well inside the VCZ zone $\left(D \gg \delta x_{0}\right)$. The behaviors in these three zones are the following:

(i) In the deep-Fresnel zone $\left(z \ll z_{\mathrm{VCZ}}\right)$, the speckles propagate as a collimated speckle beam whose average wave front is flat. Thus they broaden and overlap only because of diffraction and their longitudinal coherence properties are governed by ordinary diffraction laws (see Eq. 16 of Ref.

\footnotetext{
${ }^{1}$ In the following the subscript $n$ will denote quantities in the deep-Fresnel zone, such as the transverse and longitudinal coherence lengths $\delta x_{n}$ and $\delta z_{n}$. The subscript $f$ will label the corresponding quantities $\left(\delta x_{f}, \delta z_{f}\right)$ in the VCZ zone, where the VCZ theorem holds. This choice is done for historical reasons, being the deepFresnel and VCZ regions called also "near field" and "far field" zones, respectively.
}

[1]). As a consequence, their longitudinal size $\delta z_{n}$ is equal to the length of a collimated coherent beam of equal transversal size $\delta x_{n}$, i.e., (Eq. 17 of Ref. [1]),

$$
\delta z_{n} \sim \frac{\delta x_{n}^{2}}{\lambda} .
$$

(ii) In the Fresnel zone $\left(z_{\mathrm{VCZ}}<z<z_{\mathrm{Fr}}\right)$ the average beam wave front becomes progressively curved. The speckles diffract but at the same time start to separate because of the beam curvature. As a consequence, they take longer to overlap and their longitudinal size $\delta z_{f}$, evaluated from the distance $z$ in the outward direction of the beam, is the one expected if only diffraction would take place (Eq. 39 of Ref. $[1])$,

$$
\delta z_{f} \sim \frac{\delta x_{f}^{2}}{\lambda} \frac{1}{\left(1-z / z_{\mathrm{Fr}}\right)} .
$$

(iii) In the Fraunhofer zone $\left(z \geq z_{\mathrm{Fr}}\right)$, diffracting effects can be neglected with respect to the beam curvature, which is approximately spherical. Thus the speckles broaden at the same rate at which they spread apart, they never overlap, and behave as light pencils whose longitudinal size tends to become infinite as they propagate well inside the Fraunhofer zone (see Sec. VI in Ref. [1]).

\section{GAUSSIAN SPECKLES IN THE DEEP-FRESNEL ZONE}

In this section we first report (Sec. IV A) the analytical results worked out in Ref. [1] relative to Gaussian speckles in the deep-Fresnel zone, then (Sec. IV B) we describe the optical setup used in the measurements, and finally (Sec. IV C) we illustrate the experimental results.

\section{A. Analytical expression for $|\mu|^{2}$}

If the coherence factor characterizing the granular structure of the source has a Gaussian profile of the form

$$
C_{0}\left(\mathbf{x}_{2}-\mathbf{x}_{1}\right)=\exp \left[-\frac{\left|\mathbf{x}_{2}-\mathbf{x}_{1}\right|^{2}}{\delta x_{0}^{2}}\right],
$$

the complex coherence factor can be calculated analytically (Eq. 22 of Ref. [1]) and its squared modulus reads (Eq. 23 of Ref. [1]) 


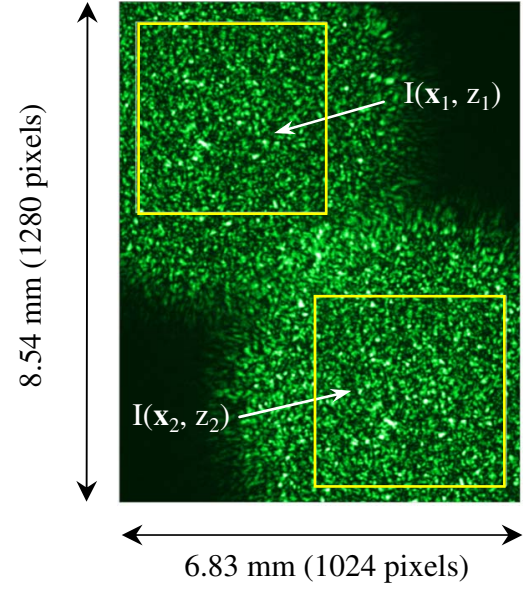

FIG. 2. (Color online) Acquisition scheme for the speckle intensity distributions at the two distances $z_{1}$ and $z_{2}$. The squares indicate the regions $(512 \times 512$ pixels $)$ that are used in the analysis. In the specific case of this figure, the two distances are the same, and, correspondingly, the two speckle intensity distributions (slightly saturated for visibility purposes) appear to be identical.

$$
\left|\mu\left(\mathbf{x}_{2}-\mathbf{x}_{1}, d z\right)\right|^{2}=\frac{1}{1+\left(d z / z_{d}\right)^{2}} \exp \left[-\frac{2\left|\mathbf{x}_{2}-\mathbf{x}_{1}\right|^{2}}{\delta x_{n}^{2}(d z)}\right],
$$

where

$$
z_{d}=\pi \delta x_{0}^{2} / \lambda
$$

and

$$
\delta x_{n}(d z)=\delta x_{0} \sqrt{1+\left(d z / z_{d}\right)^{2}} .
$$

Equations (12)-(14) show that $|\mu|^{2}$ behaves as the intensity distribution of an equivalent Gaussian beam propagating along $d z$, characterized by a beam waist equal to $\delta x_{0}$ in $d z$ $=0$. Its amplitude decays with $d z$ following a Lorentzian profile, whose width is the equivalent Rayleigh range $z_{d}$. Thus the longitudinal coherence length [defined as the distance $d z$ at which $\left.|\mu(0, d z)|^{2}=0.5\right]$ is

$$
\delta z_{n}=z_{d}=\pi \delta x_{0}^{2} / \lambda .
$$

At the same time, the width of $|\mu|^{2}$ gets larger with increasing $d z$, with a waist $\delta x_{n}(d z)$, which follows exactly the same broadening expected for a Gaussian beam [Eq. (14)].

\section{B. Setup for Gaussian speckles in the deep-Fresnel zone}

The experimental setup used for characterizing the 3D speckles in the deep-Fresnel zone is schematically shown in Fig. 1. The pseudothermal source consists of laser beam (cw He-Ne, $\lambda=0.6328 \mu \mathrm{m})$, which, after being spatially filtered $\left(F_{1}=40 \mathrm{~mm}, F_{2}=40 \mathrm{~mm}\right)$ and collimated, is shined onto a diffuser (Newport, model 20DKIT-C2, diameter $50 \mathrm{~mm}$, diffusing angle $\alpha \sim 15^{\circ}$ ), which can be rotated by means of a stepper motor. The light scattered by the diffuser is collected by a lens placed at a distance equal to its focal length $F$ $=80 \mathrm{~mm}$, and the resulting collimated light is stopped by a diaphragm of diameter $D=6.0 \mathrm{~mm}$ located at the focal plane of the lens. On this plane ( $z=0$ plane) the Fourier transform of the field emerging from the diffuser is realized and, therefore, since the intensity distribution illuminating the glass is Gaussian $I_{0}(\mathbf{x})=I_{0} \exp \left[-\mathbf{x}^{2} / w_{0}^{2}\right]$ with $\mathrm{w}_{0} \sim 3.2 \mathrm{~mm}$, the speckles formed at $z=0$ are expected to have a Gaussian coherence factor of the form of Eq. (11), with $\delta x_{0}$ $=\pi \lambda F / \mathrm{w}_{0} \sim 49 \mu \mathrm{m}$. Note that, since the beam impinging onto the diffuser is collimated, its phase profile is flat. Thus, the coherence factor $C_{0}(\mathbf{x})$ is real and the (average) phase profile of the speckle field is flat, as required for deepFresnel speckles (see Sec. II in Ref. [1]).

The intensity profile modulating this speckle field is set by the angular distribution of the light scattered by the diffuser, which, in turn, depends on the size $\rho \sim \lambda \alpha \sim 2.5 \mu \mathrm{m}$ of the grains present on its surface. This profile is reasonably flat over the diaphragm aperture because its width is $\sim \lambda F / \rho \sim 20 \mathrm{~mm}>D$. Thus, the diaphragm plane can be considered as the plane $(z=0)$ of a pseudothermal source of transversal size $D$, characterized by an average flat phase distribution with inhomogeneities of size $\delta x_{0}$. For this source the crossover between the deep-Fresnel and VCZ regimes occurs at a distance $z_{\mathrm{VCZ}} \sim D_{0} \delta x_{0} / \lambda \sim 470 \mathrm{~mm}$.

The intensity distributions $I\left(\mathbf{x}, z_{1}\right)$ and $I\left(\mathbf{x}, z_{2}\right)$ were measured, at the distances $z_{1}$ and $z_{2}$, by using a charge-coupled device (CCD) sensor (Vooskuhler, model CCD-1300, 1024 $\times 1280$ pixels, pixel size $=6.67 \times 6.67 \mu \mathrm{m}^{2}$ ) and adopting a scheme similar to a Michelson interferometer, in which the speckle beam is divided into two "twin" beams by a $30 \mathrm{~mm}$ glass cube beam splitter. In the first arm, the mirror $M_{1}$ is

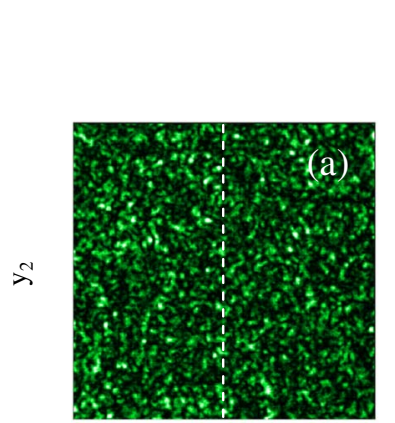

$\mathrm{X}_{2}$

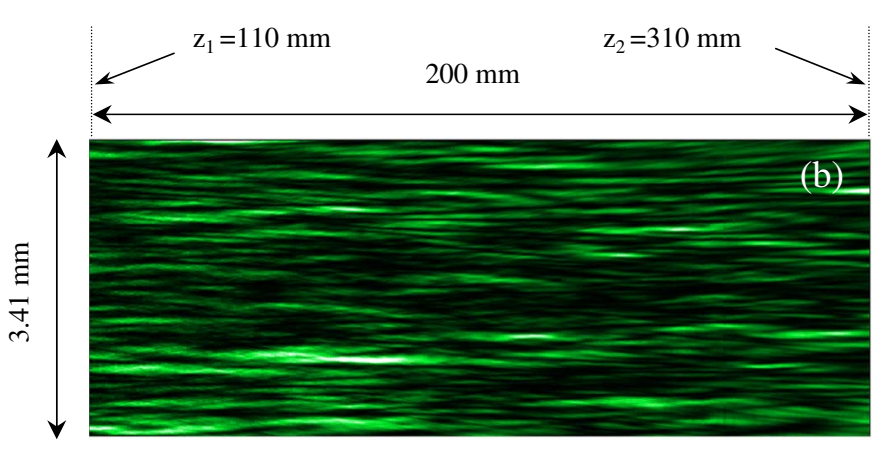

$\mathrm{dz}$

FIG. 3. (Color online) (a) $x-y$ and (b) $y-z$ intensity distributions of Gaussian speckles in the deep-Fresnel zone. The speckles look like jelly beans with a longitudinal length much longer than their transversal size, whose value $\left(\delta x_{0} \sim 49 \mu \mathrm{m}\right)$ does not change with distance. 

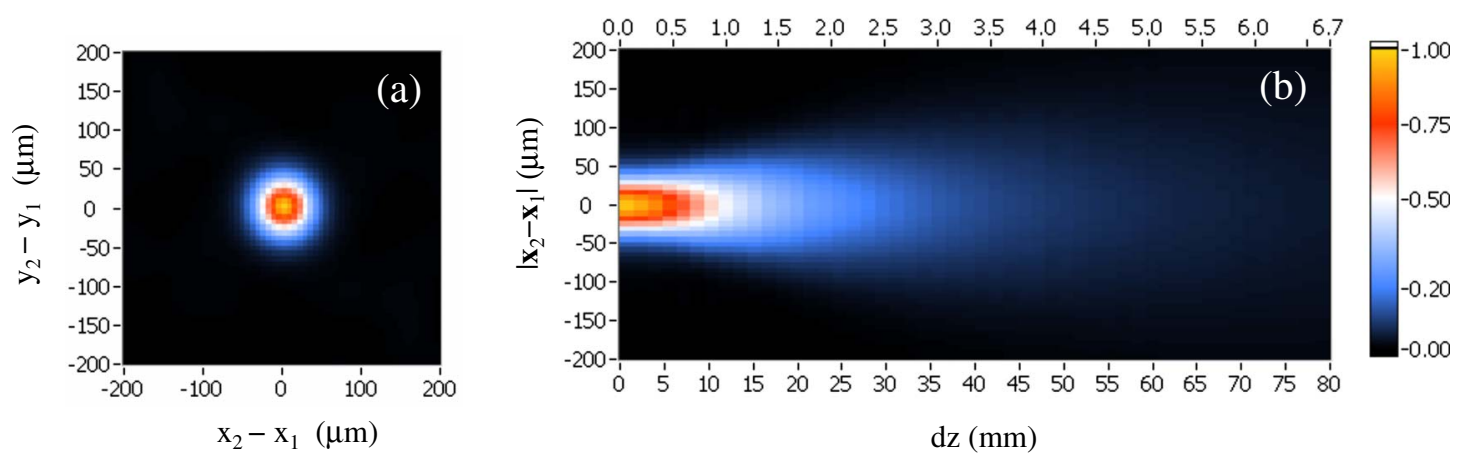

FIG. 4. (Color online) Experimental characterization of the 3D-coherence properties of Gaussian speckles in the deep-Fresnel zone; (a) transversal image of the coherence factor $\left|\mu\left(\mathbf{x}_{2}-\mathbf{x}_{1}, d z\right)\right|^{2}$ taken at $d z=0$. The speckle size is $\delta x_{0} \sim 49 \mu \mathrm{m}$. (b) Longitudinal image of the coherence factor reported as the angular averages of $\left|\mu\left(\left|\mathbf{x}_{2}-\mathbf{x}_{1}\right|, d z\right)\right|^{2}$ as a function of $d z$. Note the different transversal and longitudinal scales, showing that the longitudinal speckle size $\left(\delta z_{n} \sim 12 \mathrm{~mm}\right)$ is much larger than its transversal size, as expected from Eq. (15). The top horizontal axis in panel (b) indicates the rescaled distance $d z / z_{d}$ and allows an easy comparison with Fig. 2 of Ref. [1].

kept fixed (and slightly tilted) at an effective ${ }^{2}$ distance $z_{1}$ $=110 \mathrm{~mm}$. In the second arm, the mirror $\mathrm{M}_{2}$ is orthogonal to the optical axis and can be moved by using a computercontrolled translation stage, whose maximum travel length is $100 \mathrm{~mm}$. Under these conditions, the two speckle beams do not overlap at the CCD plane and the two intensity distributions can be acquired simultaneously within the same frame. This is illustrated in Fig. 2, where the two regions of interest $(512 \times 512$ pixels $)$ refer to the intensities measured at the two distances $z_{1}$ and $z_{2}$, i.e., $I\left(\mathbf{x}_{1}, z_{1}\right)$ (upper left square) and $I\left(\mathbf{x}_{2}, z_{2}\right)$ (lower right square). In the specific case of Fig. 2, the two distances are the same, $z_{1}=z_{2}=110 \mathrm{~mm}$, and the two beams look identical, as expected for twin speckle beams. When the optical path difference $d z=z_{2}-z_{1}$ is increased, the speckle intensity distribution measured at $z_{2}$ starts to change and becomes progressively uncorrelated with respect to the one detected at $z_{1}$.

\section{Experimental results}

The first experimental characterization was to investigate the loss of correlation with $d z$. This feature is qualitatively illustrated in Fig. 3, where the transversal $x-y$ speckle intensity distribution $I\left(\mathbf{x}, z_{2}\right)$ is shown in panel (a) for $z_{2}$ $=110 \mathrm{~mm}$, and its longitudinal $y-z$ distribution is reported in panel (b) as a function of $d z$. Figure 3 was obtained with the diffuser still and changing $z_{2}$ in steps of $0.8 \mathrm{~mm}$ from 110 to $310 \mathrm{~mm}$. For each $I\left(\mathbf{x}_{2}, z_{2}\right)$, the $y$ cut was taken at the center of the distribution as indicated by the vertical dotted line in panel (a).

The analysis of the 3D speckle coherence properties was carried out by cross correlating the distributions $I\left(\mathbf{x}_{1}, z_{1}\right)$ and

\footnotetext{
${ }^{2}$ The effective distance is the geometrical distance reduced by an offset distance $\Delta z$, which takes into account the (double) propagation of light inside the cube beam splitter. In the limit of small angles, this offset is given by $\Delta z=2 L(n-1) / n, L$ being the beam splitter side and $n$ being the glass refraction index. For $L=30 \mathrm{~mm}$ and $n=1.5, \Delta z=20 \mathrm{~mm}$. All the distances reported in this paper are to be interpreted as effective distances.
}

$I\left(\mathbf{x}_{2}, z_{2}\right)$ as a function of $d z=z_{2}-z_{1}$. For each $d z$ the diffuser was rotated and the cross correlation was averaged over several speckle configurations. The results are shown in Fig. 4, in which we report the transversal $\left|\mu\left(\mathbf{x}_{2}-\mathbf{x}_{1}, d z=0\right)\right|^{2}$ [panel (a)] and longitudinal $\left|\mu\left(\left|\mathbf{x}_{2}-\mathbf{x}_{1}\right|, d z\right)^{2}\right|$ [panel (b)] behaviors of the coherence factor. In panel (b) the top horizontal axis shows also the rescaled $d z / z_{d}$ distance for an easy comparison with Fig. 2 of Ref. [1]. One may notice the different scales on the horizontal and vertical axes, showing that the longitudinal length of the speckles is much larger than their transversal size. This happens, consistently with Eq. (10) or (15), whenever the transverse speckle size is much larger than the wavelength $\left(\delta x_{0} \gg \lambda\right)$.

A quantitative analysis of the features illustrated in Fig. 4 can be appreciated in Fig. 5, where we report the transversal (angularly averaged) profiles of $\left|\mu\left(\left|\mathbf{x}_{2}-\mathbf{x}_{1}\right|, d z\right)^{2}\right|$ as a function of the radial distance $\left|\mathbf{x}_{2}-\mathbf{x}_{1}\right|$ at various $d z$. [panel (a)] and the longitudinal profile $\left|\mu\left(\mathbf{x}_{2}-\mathbf{x}_{1}=0, d z\right)\right|^{2}$ taken in correspondence of its maximum $\left(\mathbf{x}_{2}=\mathbf{x}_{1}\right)$. Figure 5(a) shows that, as predicted by Eq. (12), the radial profiles are Gaussians with the highest curve $(d z=0)$ providing the estimate of the transversal speckle size, i.e., $\delta x_{0} \sim 49 \mu \mathrm{m}$. As $d z$ increases, the radial profiles become broader and lower but conserve the 2D area, as predicted by Eq. 18 of Ref. [1]. For the sake of clarity, the comparison with theory [Eq. (12)] is shown, as a solid curve, only in the case of $d z=15 \mathrm{~mm}$. The longitudinal profile of $|\mu|^{2}$ taken at its peak value $\left(\mathbf{x}_{2}=\mathbf{x}_{1}\right)$ is reported as a function of $d z$ in Fig. 5(b). Also in this case, the Lorentzian theoretical profile predicted by Eq. (12) (solid curve) is well matched by the experimental data (circles) and the longitudinal length of the speckles, recovered as the value of $d z$ for which $|\mu|^{2}=0.5$ is $\delta z_{n} \sim 12.0 \mathrm{~mm}$, in good agreement with the predictions of Eq. (15).

\section{GAUSSIAN SPECKLES IN THE VCZ ZONE}

As for Sec. IV C, we first (Sec. V A) report the analytical results worked out in Ref. [1] relative to Gaussian speckles in the VCZ zone, then (Sec. V B) we describe the optical setup used in the measurements, and finally we illustrate the 

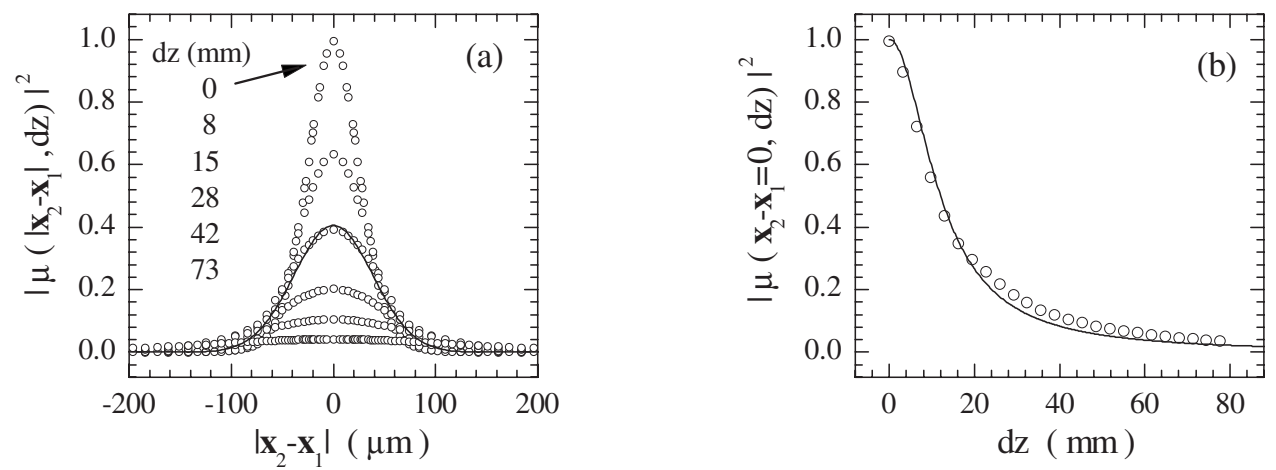

FIG. 5. Behavior of the 3D-coherence factor for Gaussian speckles in the deep-Fresnel zone: (a) radial profiles $\left|\mu\left(\left|\mathbf{x}_{2}-\mathbf{x}_{1}\right|, d z\right)\right|^{2}$ at different $d z$ values (circles). The highest curve $(d z=0)$ allows us to estimate the speckle size $\delta x_{0} \sim 49 \mu \mathrm{m}$. As $d z$ increases the curves become progressively lower and broader. For the sake of clarity, comparison with theory (solid curve) is shown only for $d z=15 \mathrm{~mm}$. (b) Longitudinal profile $\left|\mu\left(\mathbf{x}_{2}-\mathbf{x}_{1}=0, d z\right)\right|^{2}$ taken at its peak value $\left(\mathbf{x}_{2}=\mathbf{x}_{1}\right)$ as a function of $d z$ (circles) and comparison with theory (solid curve). The longitudinal speckle size, defined as the distance at which $|\mu|^{2}=0.5$, is $\delta z_{n} \sim 12 \mathrm{~mm}$.

experimental results far from the Fraunhofer zone (Sec. V C) and inside the Fraunhofer zone (Sec. V D).

\section{A. Analytical expression for $|\mu|^{2}$}

In this zone the results are, to a good approximation, independent of the microscopic details of the source, which, therefore, will be not taken into account. Assuming a Gaussian profile for the average intensity distribution at the source plane,

$$
I_{0}(\mathbf{x})=I_{0} \exp \left[-\frac{\mathbf{x}^{2}}{D^{2}}\right],
$$

the squared modulus of the coherence factor $\mu$ reads (Eq. 23 of Ref. [1])

$$
\left|\mu\left(\mathbf{x}_{1}, z_{1} ; \mathbf{x}_{2}, z_{1}+d z\right)\right|^{2}=\frac{1}{1+\gamma^{2}\left(z_{1}, d z\right)} \exp \left[-\frac{2\left|\mathbf{x}_{2} \frac{z_{1}}{z_{2}}-\mathbf{x}_{1}\right|^{2}}{\delta x_{f}^{2}\left(z_{1}, d z\right)}\right],
$$

in which $z_{2}=z_{1}+d z$ and

$$
\begin{gathered}
\gamma\left(z_{1}, d z\right)=\frac{\pi D^{2}}{\lambda z_{1}} \frac{d z}{z_{1}+d z}, \\
\delta x_{f}\left(z_{1}, d z\right)=\delta x_{f}\left(z_{1}, 0\right) \sqrt{1+\gamma^{2}\left(z_{1}, d z\right)},
\end{gathered}
$$

$$
\delta x_{f}\left(z_{1}, 0\right)=\frac{\lambda}{\pi D} z_{1} .
$$

Equations (17) and (18) show that $|\mu|^{2}$ exhibits its maximum amplitude at $d z=0$, where $\gamma\left(z_{1}, d z=0\right)=0$. In accordance with the VCZ theorem, the speckle size is given by Eq. (18c), namely, $\delta x_{f}\left(z_{1}, 0\right)=(\lambda / \pi D) z_{1}$. As $d z$ increases, $|\mu|^{2}$ undergoes a decay which is controlled by the parameter $\gamma\left(z_{1}, d z\right)$, whose asymptotic $(d z \rightarrow \infty)$ value is $\pi D^{2} / \lambda z_{1}$. Accordingly, the asymptotic value for the amplitude of $|\mu|^{2}$, evaluated along the directions that define the radial broadening of the beam $\mathbf{x}_{2}\left(z_{1} / z_{2}\right)=\mathbf{x}_{1}$, is

$$
\lim _{d z \rightarrow \infty}\left|\mu\left(\mathbf{x}_{1}, z_{1} ; \mathbf{x}_{2}=\frac{z_{1}}{z_{2}} \mathbf{x}_{1}, z_{1}+d z\right)\right|^{2}=\frac{1}{1+\left(z_{\mathrm{Fr}} / z_{1}\right)^{2}},
$$

where we have introduced the Fraunhofer distance defined, in the case of Gaussian speckles, as

$$
z_{\mathrm{Fr}}=\frac{\pi D^{2}}{\lambda}
$$

Equation (19) shows that the asymptotic decay value of $|\mu|^{2}$ can approach zero only when $z_{1} \ll z_{\mathrm{Fr}}$, i.e., when we are well inside the Fresnel zone $\left(z_{\mathrm{VCZ}}<z_{1}<z_{\mathrm{Fr}}\right)$. Under these circumstances we can define the longitudinal coherence length $\delta z_{f}$ of the speckles as the distance at which $|\mu|^{2}=0.5$. This con-

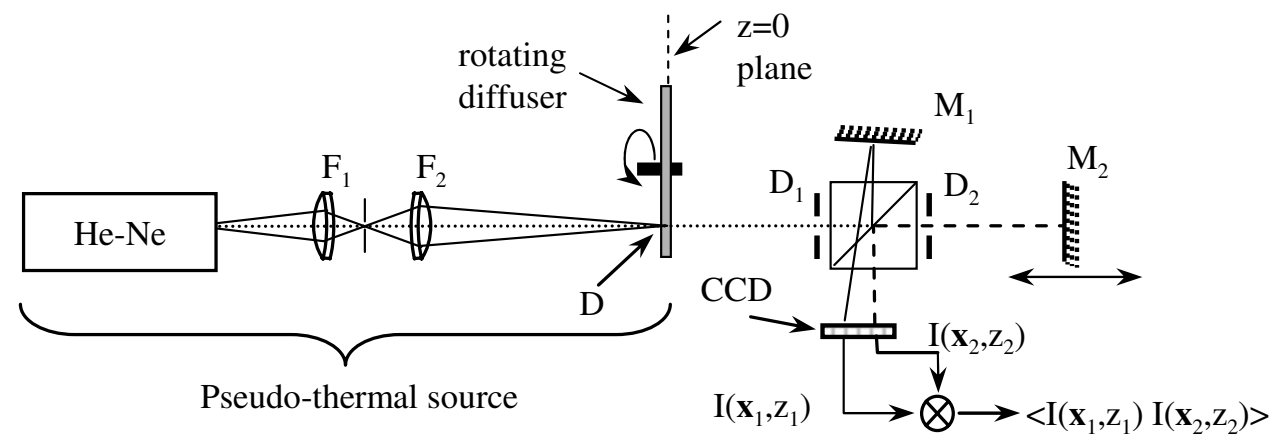

FIG. 6. Schematic diagram of the setup used for studying 3D Gaussian speckles in the VCZ region. 
dition, which corresponds to setting $\gamma=1$ in Eq. (18a), reads as

$$
\delta z_{f}=z_{d} \frac{1}{\left(1-z_{1} / z_{\mathrm{Fr}}\right)} \quad\left(z_{1}<z_{\mathrm{Fr}}\right),
$$

in which $z_{d}$, defined as

$$
z_{d}=\frac{\pi \delta x_{f}^{2}\left(z_{1}, 0\right)}{\lambda},
$$

corresponds to the longitudinal length of a speckle of transversal size $\delta x_{f}\left(z_{1}, 0\right)$ that would propagate only because of diffraction. Thus Eq. (21) shows that Gaussian speckles in the VCZ zone, relatively far from the Fraunhofer distance $\left(z_{1}<z_{\mathrm{Fr}}\right)$, are characterized by a longitudinal length that can be much larger (as $z_{1}$ approach $z_{\mathrm{Fr}}$ ) than the length exhibited by speckles of the same transversal size but propagating inside the deep-Fresnel zone.

On the other hand, when we are in the Fraunhofer zone $\left(z_{1} \gg z_{\mathrm{Fr}}\right)$, Eq. (19) shows that $|\mu|^{2}$ never decays to a value sensibly different from unity. Therefore, the longitudinal coherence persists forever, and the speckles tend to become infinitely long.

\section{B. Setup for Gaussian speckles in the VCZ zone}

The experimental setup used for characterizing the 3D speckles in the VCZ zone is schematically shown in Fig. 6. The pseudothermal source is similar to the one shown in Fig. 1 , but in this case the Gaussian beam is focused onto the diffuser and there is no Fourier lens beyond the diffuser. Thus the $z=0$ plane of the thermal source corresponds to the diffuser plane. The remaining part of the setup (beam splitter, mirrors, and CCD) is identical to one described in Fig. 1, with the exception of the diaphragms $D_{1}$ and $D_{2}$ placed along the optical axis before and after the beam splitter. The first diaphragm $D_{1}=3.0 \mathrm{~mm}$ is used to limit the size of the first speckle beam at the CCD plane, while the second diaphragm $D_{2}=5.0 \mathrm{~mm}$ limits the size of the second speckle beam that, propagating over a distance $z_{2}>z_{1}$ (maximum value of $z_{2} / z_{1} \sim 2$ ), would otherwise become too large and overlap to the first speckle beam. We have checked by ray tracing and experimentally verified that the presence of these two diaphragms does not affect at all the speckle beam propagation. The setup described in Fig. 6 has been used for carrying out two experiments in which, by changing the size of the beam onto the diffuser, the $3 \mathrm{D}$ coherences far from and inside the Fraunhofer zones were investigated.

\section{VCZ regime far from the Fraunhofer zone $\left(z_{1} \sim 0.41 z_{\mathrm{Fr}}\right)$}

In the first experiment the Gaussian beam was focused onto the diffuser to a diameter $D \sim 306 \mu \mathrm{m}$ [see Eq. (16)], so that the crossover distance between the deep-Fresnel and $\mathrm{VCZ}$ regimes occurs at $z_{\mathrm{VCZ}} \sim D \rho / \lambda \sim 1.2 \mathrm{~mm}(\rho=2.5 \mu \mathrm{m}$, see Sec. IV B). The corresponding Fraunhofer distance is $z_{\mathrm{Fr}} \sim \pi D^{2} / \lambda \sim 465 \mathrm{~mm}$. The fixed distance was $z_{1}$ $=192 \mathrm{~mm}$ so that $z_{1} \sim 0.41 z_{\mathrm{Fr}}$ and $z_{2}$ was varied from $z_{2}$ $=z_{1}$ to $z_{2}=392 \mathrm{~mm} \sim 0.81 z_{\mathrm{Fr}}$. In this way we spanned a range of distances $d z$ where the speckle coherence length $\delta z_{f}$

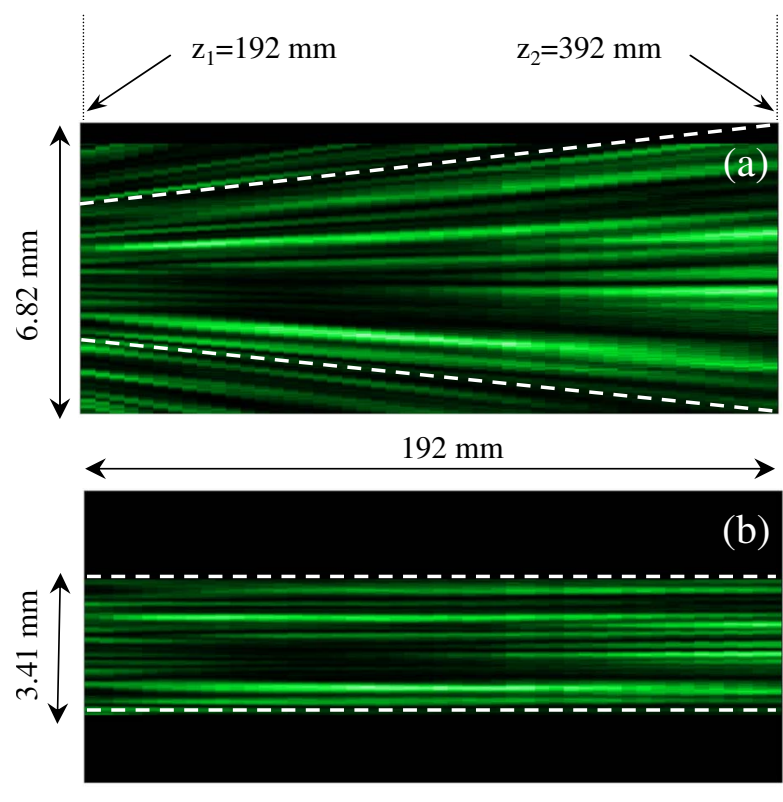

$\mathrm{dz}$

FIG. 7. (Color online) Panel (a): $y-z$ intensity distribution of Gaussian speckles in the VCZ zone; as the speckles propagate along $z$, they become larger and spread apart along the radial directions indicated by the two diverging dashed lines. Panel (b): $y$ - $z$ intensity distribution after rescaling the transversal coordinate for its radial broadening, i.e., $y^{\prime}=\left(z_{1} / z_{2}\right) y$; the rescaled speckles propagate along $z$ maintaining the same size. The parallel dashed lines delimit the rescaled region, which corresponds to the region of panel (a) inside the diverging lines.

is expected to be sensibly longer than the length $z_{d}$ associated to pure diffracting speckles of the same transverse size [see Eqs. (21) and (22)]. The latter one is $z_{d} \sim 79 \mathrm{~mm}$ and the transverse speckle size at $z_{1}$ is $\delta x_{f}\left(z_{1}\right) \sim 127 \mu \mathrm{m}$ [Eq. (18c)]. Figure 7(a) reports the $y-z$ cut of the intensity speckle distribution as a function of $d z=z_{2}-z_{1}$. This figure was obtained with the diffuser still, blocking the first speckle beam, using the entire CCD frame, and changing $z_{2}$ in steps of $0.8 \mathrm{~mm}$ from $192 \mathrm{~mm}$ to $392 \mathrm{~mm}$.

Differently from what happens in the deep-Fresnel zone where the speckles maintain the same size regardless of the distance $d z$ [see Fig. 3(b)], in this case, as $d z$ increases, the speckles become larger and spread apart along the radial directions indicated by the two diverging dashed lines reported in Fig. 7(a). This is exactly what predicted by the VCZ theorem, according to which the speckle size increases linearly with the propagation distance $z_{2}$. Thus we can rescale the transverse coordinate $y$ by the factor $\left(z_{1} / z_{2}\right)$ and replot Fig. 7 (a) by using on the vertical axis the rescaled coordinate $y^{\prime}=\left(z_{1} / z_{2}\right) y$. The result is shown in Fig. 7(b), where the rescaled speckles propagate along $z_{2}$ maintaining the same size. The parallel dashed lines reported in Fig. 7(b) delimit the rescaled region, which corresponds to the region panel (a) inside the diverging lines.

Due to the enlargement of the speckles with distance, the analysis of the 3D-coherence properties of the speckle field was carried out differently from what was done in the case of deep-Fresnel speckles. First of all, we rescaled the transver- 

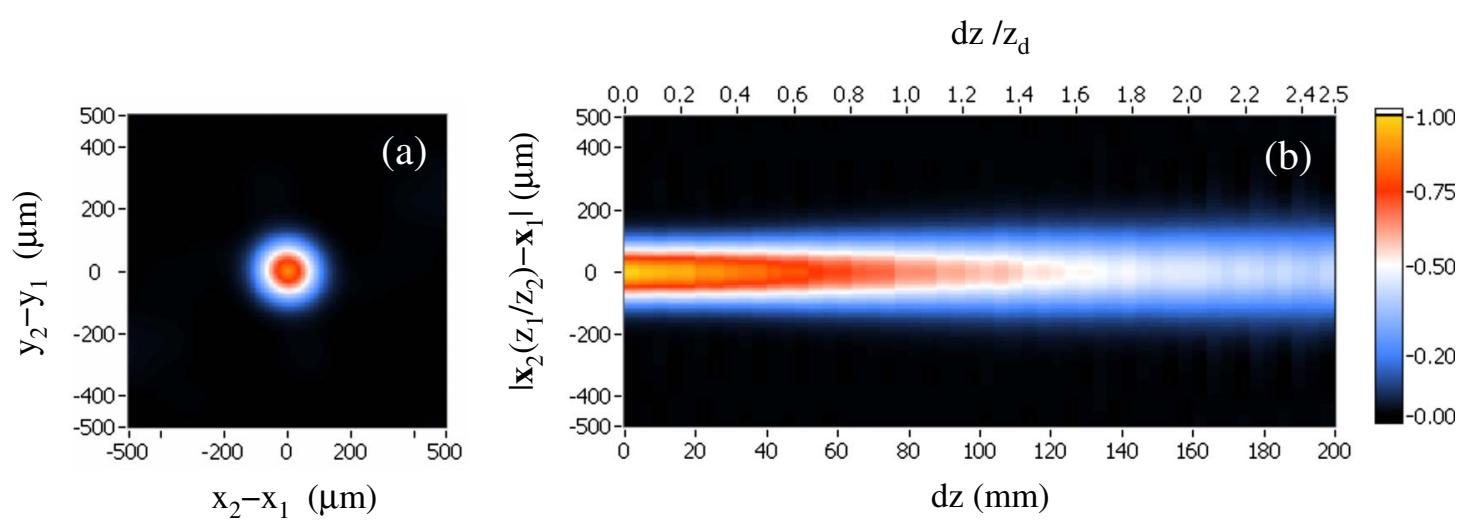

FIG. 8. (Color online) Experimental characterization of the 3D-coherence properties of Gaussian speckles in the VCZ region, far from the Fraunhofer zone $\left(z_{1} / z_{\mathrm{Fr}} \sim 0.4\right)$ : (a) transversal image of the coherence factor $\left|\mu\left[\mathbf{x}_{2}\left(z_{1} / z_{2}\right)-\mathbf{x}_{1}, d z\right]\right|^{2}$ taken at $d z=z_{2}-z_{1}=0$. The speckle size at $z_{1}$ is $\delta x_{f}\left(z_{1}\right) \sim 127 \mu \mathrm{m}$. (b) Longitudinal image of the coherence factor reported as the angular average of $\left|\mu\left[\mathbf{x}_{2}\left(z_{1} / z_{2}\right)-\mathbf{x}_{1}, d z\right]\right|^{2}$ as a function of $d z$. The speckle length is $\delta z_{f} \sim 135 \mathrm{~mm}$. The top horizontal axis in panel (b) indicates the rescaled distance $d z / z_{d}$, allowing an easy comparison with Fig. 2 of Ref. [1] and with Fig. 4 of this paper.

sal coordinates of the intensity distributions at $z_{2}$ by the factor $z_{1} / z_{2}$. In this way we obtained the rescaled distribution $I^{\prime}\left(\mathbf{x}_{2}^{\prime}, z_{2}\right)=I\left(\left[z_{1} / z_{2}\right] \mathbf{x}_{2}, z_{2}\right)$ which now extends over a transversal region reduced by the factor $z_{1} / z_{2}$. Operatively, we carried out this task by resampling (and interpolating) $I\left(\mathbf{x}_{2}, z_{2}\right)$ with a number of pixels reduced by the factor $z_{1} / z_{2}$ with respect to the number of its physical pixels. Then we selected a subset of $I\left(\mathbf{x}_{1}, z_{1}\right)$ corresponding to the same transversal region of $I^{\prime}\left(\mathbf{x}_{2}^{\prime}, z_{2}\right)$ so that the number of pixels is equal in the two images. At this point we cross correlated $I\left(\mathbf{x}_{1}, z_{1}\right)$ with $I^{\prime}\left(\mathbf{x}_{2}^{\prime}, z_{2}\right)$ and obtained a cross-correlation function in terms of the transversal variable $\mathbf{x}_{2}^{\prime}-\mathbf{x}_{1}=\left(z_{1} / z_{2}\right) x_{2}$ $-x_{1}$. This is shown in Fig. 8, in which we report the transversal $\left|\mu\left(\mathbf{x}_{2}^{\prime}-\mathbf{x}_{1}, d z=0\right)\right|^{2} \quad$ [panel (a)] and longitudinal $\left|\mu\left(\mathbf{x}_{2}^{\prime}=\mathbf{x}_{1}, d z\right)\right|^{2}$ [panel (b)] behaviors of the rescaled coherence factor. As in Fig. 4, the (top) horizontal axis indicates the rescaled $d z / z_{d}$ distance for an easy comparison with Fig. 2 of Ref. [1] and with Fig. 4 of this paper. From the latter one, we observe that in the VCZ regime the speckle length is sensibly longer than what expected for pure diffraction: in-

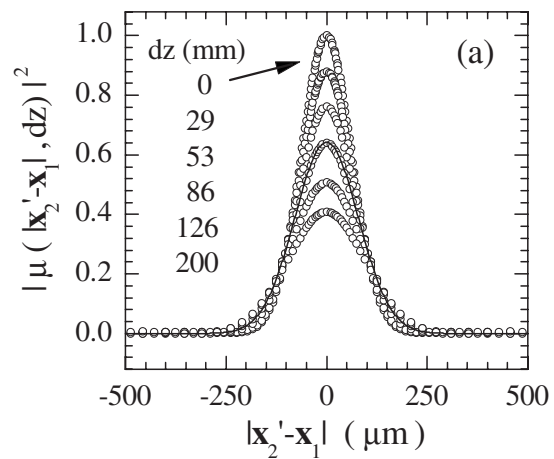

deed, while in Fig. 4(b) a coherence level of 0.5 (white color) occurs at $d z / z_{d} \sim 1$ [see also Eq. (12)], in the case of Fig. 8 (b), this takes place at $d z / z_{d} \sim 1.7$, consistently with what predicted by Eq. (19) when $z_{1} / z_{\mathrm{Fr}}=0.41$.

A quantitative analysis of the features described in Fig. 8 can be appreciated in Fig. 9, where, similarly to what was done for Fig. 5, we report the transversal (angularly averaged) profiles of $\left|\mu\left(\left|\mathbf{x}_{2}^{\prime}-\mathbf{x}_{1}\right|, d z\right)\right|^{2}$ as a function of $\left|\mathbf{x}_{2}^{\prime}-\mathbf{x}_{1}\right|$ at various $d z$ [panel (a)] and the longitudinal profile $\mid \mu\left(\mathbf{x}_{2}^{\prime}-\mathbf{x}_{1}\right.$ $=0, d z)\left.\right|^{2}$ taken in correspondence of its maximum $\left(\mathbf{x}_{2}^{\prime}=\mathbf{x}_{1}\right)$. Figure 9(a) shows that, as predicted by Eq. (17), the (transversally rescaled) radial profiles are Gaussians with the highest curve $(d z=0)$ providing the estimate of the transversal speckle size at $z_{1}$, i.e., $\delta x_{f} \sim 127 \mu \mathrm{m}$. For the sake of clarity, the comparison with theory [Eq. (17)] is shown, as a solid curve, only for the case of $d z=86 \mathrm{~mm}$. As $d z$ increases, the radial profiles become increasingly broader and lower, but, differently from Fig. 5, their amplitudes never vanish. This feature is clearly shown in Fig. 9(b), where the amplitude of $|\mu|^{2}$ tends asymptotically $(d z \rightarrow \infty)$ to the value 0.16 (indi-

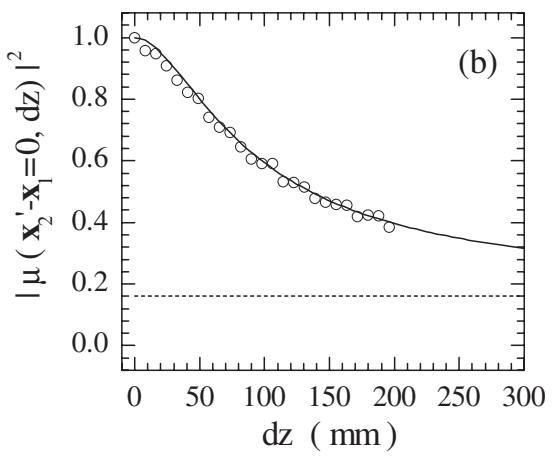

FIG. 9. Behavior of the 3D-coherence factor for Gaussian speckles in the VCZ region, far from the Fraunhofer zone $\left(z_{1} / z_{\mathrm{Fr}} \sim 0.4\right)$ : (a) radial profiles $\left|\mu\left(\mathbf{x}_{2}^{\prime}-\mathbf{x}_{1}, d z\right)\right|^{2}$ as a function of the rescaled difference $\mathbf{x}_{2}^{\prime}-\mathbf{x}_{1}=\left(z_{1} / z_{2}\right) \mathbf{x}_{2}-\mathbf{x}_{1}$ at various $d z=z_{2}-z_{1}$ $\left(z_{1}=192 \mathrm{~mm}\right.$, fixed). The highest curve $(d z=0)$ allows us to estimate the speckle size $\delta x_{f} \sim 127 \mu \mathrm{m}$. For the sake of clarity, comparison with theory (solid curve) is shown only for $d z=86 \mathrm{~mm}$; (b) longitudinal profile $\left|\mu\left(\mathbf{x}_{2}^{\prime}-\mathbf{x}_{1}=0, d z\right)\right|^{2}$ taken at its peak value $\left(\mathbf{x}_{2}^{\prime}=\mathbf{x}_{1}\right)$ as a function of $d z$ (circles) and comparison with theory (solid curve). The longitudinal speckle size, defined as the distance at which $|\mu|^{2}$ $=0.5$, is $\delta z_{f} \sim 135 \mathrm{~mm} \sim 1.7 z_{d}$. The dashed line indicates the asymptotic $(d z \rightarrow \infty)$ value of $|\mu|^{2}$ [see Eq. (19)]. 

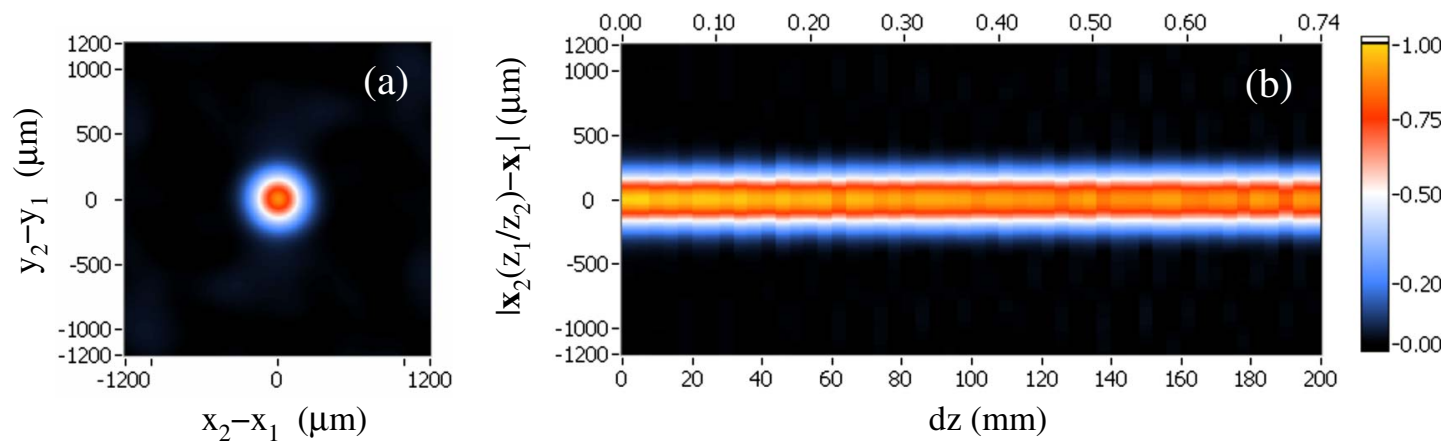

FIG. 10. (Color online) Experimental characterization of the 3D-coherence properties of Gaussian speckles in the VCZ region, inside the Fraunhofer zone $\left(z_{1} / z_{\mathrm{Fr}} \sim 1.40\right)$ : (a) transversal image of the coherence factor $\left|\mu\left[\mathbf{x}_{2}\left(z_{1} / z_{2}\right)-\mathbf{x}_{1}, d z\right]\right|^{2}$ taken at $d z=z_{2}-z_{1}=0$. The speckle size at $z_{1}$ is $\delta x_{f}\left(z_{1}\right) \sim 233 \mu \mathrm{m}$. (b) Longitudinal image of the coherence factor reported as the radial average of $\left|\mu\left[\mathbf{x}_{2}\left(z_{1} / z_{2}\right)-\mathbf{x}_{1}, d z\right]\right|^{2}$ as a function of $d z$. The top horizontal axis in panel (b) indicates the rescaled distance $d z / z_{d}$, allowing an easy comparison with Fig. 2 of Ref. [1] and with Figs. 4 and 8 of this paper.

cated by the dashed line), which is consistent with Eq. (19) $\left(z_{1} / z_{\mathrm{Fr}} \sim 0.4\right)$. The data (open symbols) reported in Fig. 9(b) match quite accurately the expected theoretical behavior [solid curve, Eq. (17)] and show that longitudinal speckle length (defined as the $z$ value for which $|\mu|^{2}=0.5$ ) is $\delta z_{f}$ $\sim 135 \mathrm{~mm}$, a value noticeably larger $\left(d z \sim 1.7 z_{d}\right)$ than the one expected from pure diffraction.

\section{VCZ regime inside the Fraunhofer zone $\left(z_{1} \sim 1.40 z_{\mathrm{Fr}}\right)$}

In the second experiment the Gaussian beam was focused to a diameter $D \sim 166 \mu \mathrm{m}$, so that the Fraunhofer distance was $z_{\mathrm{Fr}} \sim \pi D^{2} / \lambda \sim 137 \mathrm{~mm}$. The fixed distance was $z_{1}$ $=192 \mathrm{~mm}$ so that $z_{1} \sim 1.40 z_{\mathrm{Fr}}$ and $z_{2}$ was varied from $z_{2}$ $=z_{1}$ to $z_{2}=392 \mathrm{~mm} \sim 2.86 z_{\mathrm{Fr}}$. The transverse speckle size at $z_{1}$ was $\delta x_{f}\left(z_{1}\right) \sim 233 \mu \mathrm{m}$ and the correspondent pure diffraction length was $z_{d} \sim 270 \mathrm{~mm}$. The analysis was carried out by following the same procedure described in Sec. V B, i.e., by rescaling the transversal coordinates of the intensity distributions at $z_{2}$ by the factor $z_{1} / z_{2}$. The transversal and longitudinal coherence factors obtained in this way are shown in Fig. 10, in which we adopted the same color scale of Fig. 8. By comparing these two figures we notice that, while the transversal coherences [panel (a)] appear to be identical (aside for a scale factor given by the ratio of the speckle sizes in the two cases), the longitudinal ones are fairly different. In Fig. 8(b), the longitudinal speckle is "closed," meaning that a correlation level of 0.5 (corresponding to the white color) is contoured by a closed curve, whose maximum extension stretches up to $\sim 135 \mathrm{~mm}\left(d z / z_{d} \sim 1.7\right)$. Conversely, in Fig. $10(\mathrm{~b})$, all the curves corresponding to coherence levels of
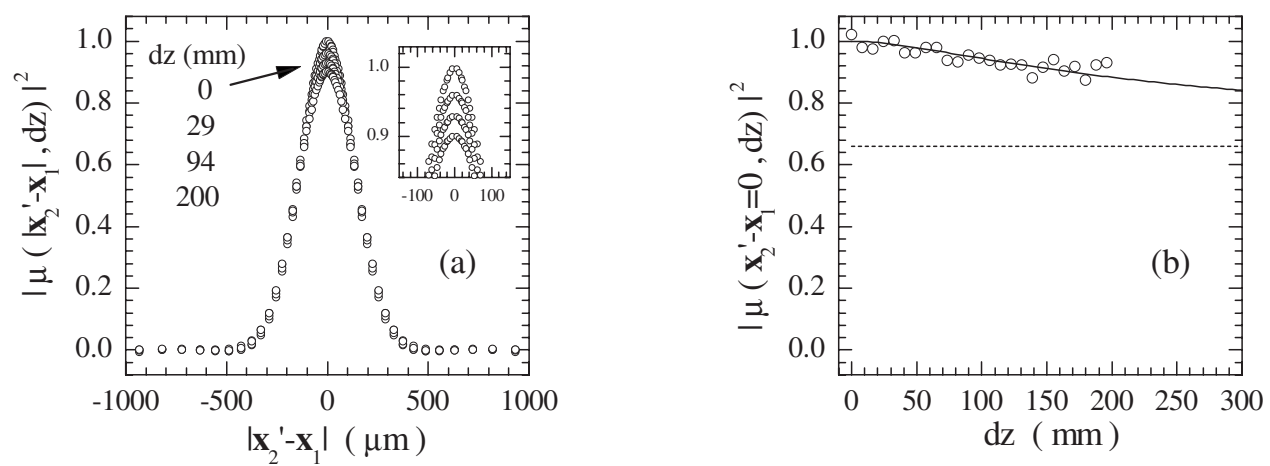

FIG. 11. Behavior of the 3D-coherence factor for Gaussian speckles in the VCZ region inside the Fraunhofer zone $\left(z_{1} / z_{\mathrm{Fr}} \sim 1.40\right)$ : (a) radial profiles $\left|\mu\left(\mathbf{x}_{2}^{\prime}-\mathbf{x}_{1}, d z\right)\right|^{2}$ as a function of the rescaled difference $\mathbf{x}_{2}^{\prime}-\mathbf{x}_{1}=\left(z_{1} / z_{2}\right) \mathbf{x}_{2}-\mathbf{x}_{1}$ at various $d z=z_{2}-z_{1}$. $\left(z_{1}=192 \mathrm{~mm}\right.$, fixed). The highest curve $(d z=0)$ allows us to estimate the speckle size $\delta x_{f} \sim 233 \mu \mathrm{m}$. The inset shows the slight differences

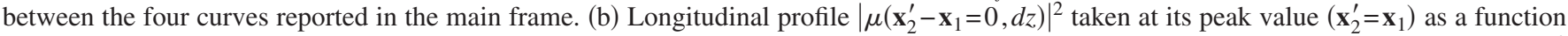
of $d z$ (circles) and comparison with theory (solid curve). The longitudinal speckle size cannot be defined because, in this case, the profile $|\mu|^{2}$ never attain a level lower than 0.66, indicated by the dashed line [see Eq. (19)]. 


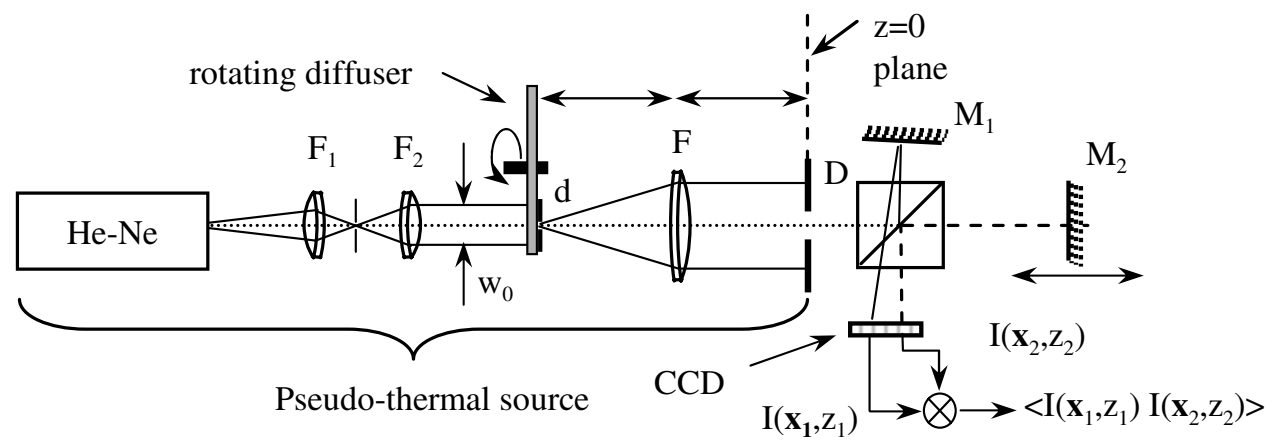

FIG. 12. Schematic diagram of the setup used for studying 3D Airy speckles in the deep-Fresnel region.

$\sim 0.8$, or higher, appear to be open and suggest that speckles are much longer than the maximum distance scanned in Fig. 10(b). This behavior is confirmed quantitatively in Fig. 11(a), where all the transverse profiles $\left|\mu\left(\mathbf{x}_{2}^{\prime}-\mathbf{x}_{1}=0, d z\right)\right|^{2}$ at various $d z$ [panel (a)] are characterized by almost the same amplitude close to unity. Similarly, Fig. 11(b) shows that the onaxis longitudinal profile $\left|\mu\left(\mathbf{x}_{2}^{\prime}-\mathbf{x}_{1}=0, d z\right)\right|^{2}$ remains substantially constant $(\sim 10 \%$ decay $)$ over the entire range of spanned distances up to $d z=200 \mathrm{~mm}$. As for Fig. 9(b), the data (open symbols) follow accurately the theoretical behavior (solid curve), but in this case the asymptotic decay value is so high $\left[|\mu(d z \rightarrow \infty)|^{2}=0.66\right.$; see dashed line $]$ that it would be fairly misleading to talk about a speckle length. On the contrary, the longitudinal coherence seems to persist forever, and the speckles tend to become infinitely long as $z_{1} / z_{\mathrm{Fr}} \rightarrow \infty$.

\section{AIRY SPECKLES IN THE DEEP-FRESNEL ZONE}

This sections aims at characterizing the 3D-coherence properties of Airy speckles in the deep-Fresnel zone. Airy speckles, i.e., speckles characterized by coherence factor equal to the Airy function, are usually observed in the VCZ region and are produced by illuminating a diffuser with a plane-wave whose transversal size is limited by a circular aperture. However, it is also possible to observe them in the deep-Fresnel zone by following the same method described in Sec. IV and realized with the setup reported in Fig. 12. This setup is identical to the one reported in Fig. 1, except for the much larger collimated beam impinging on to diffuser $\left(w_{0} \sim 20 \mathrm{~mm}\right)$ and for the presence of a pinhole of diameter $d=1.5 \mathrm{~mm}$ placed right after the diffuser. The pinhole selects a very small portion of the beam, so that the average
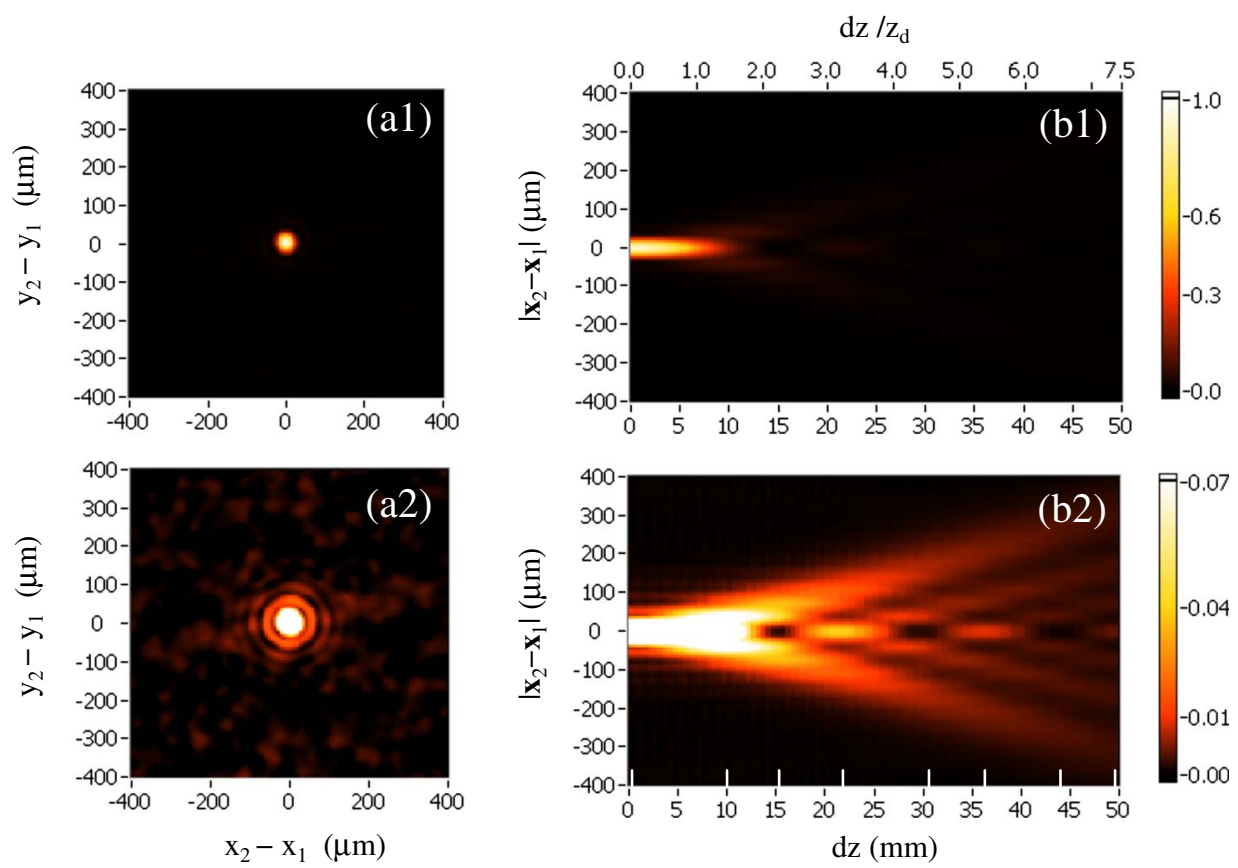

FIG. 13. (Color online) Experimental characterization of the 3D-coherence properties of Airy speckles in the deep-Fresnel zone; [(a1) and (a2)] transversal images of the coherence factor $\left|\mu\left(\mathbf{x}_{2}-\mathbf{x}_{1}, d z\right)\right|^{2}$ taken at $d z=0$ at two different intensity-scale levels. Image (a2) is highly saturated to enhance the rings visibility. The speckle size is $\delta_{x_{0}}^{\mathrm{FWHM}} \sim 36 \mu \mathrm{m}$. [(b1) and (b2)] Longitudinal images of the coherence factor reported as radial averages of $\left|\mu\left(\left|\mathbf{x}_{2}-\mathbf{x}_{1}\right|, d z\right)\right|^{2}$ as a function of $d z$ at the same intensity-scale levels of panels (a1) and (a2). Image (b2) shows the neat complex 3D structure of the coherence factor, with the presence of many minima and maxima (indicated by the white vertical segments) alternating on axis $\left(\mathbf{x}_{2}=\mathbf{x}_{1}\right)$. The top horizontal axis in panel (b) indicates the rescaled distance $d z / z_{d}$. 

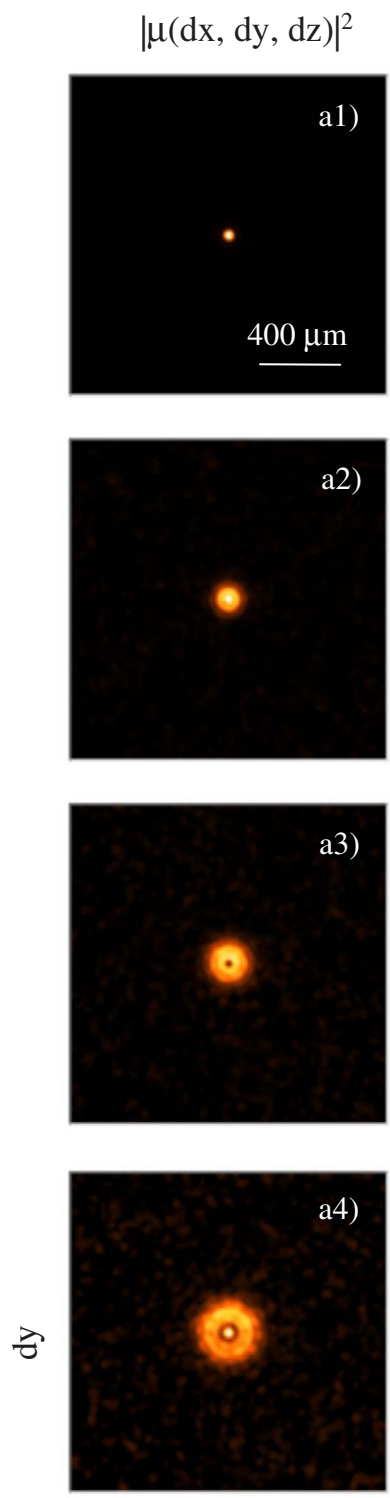

$\mathrm{dx}$
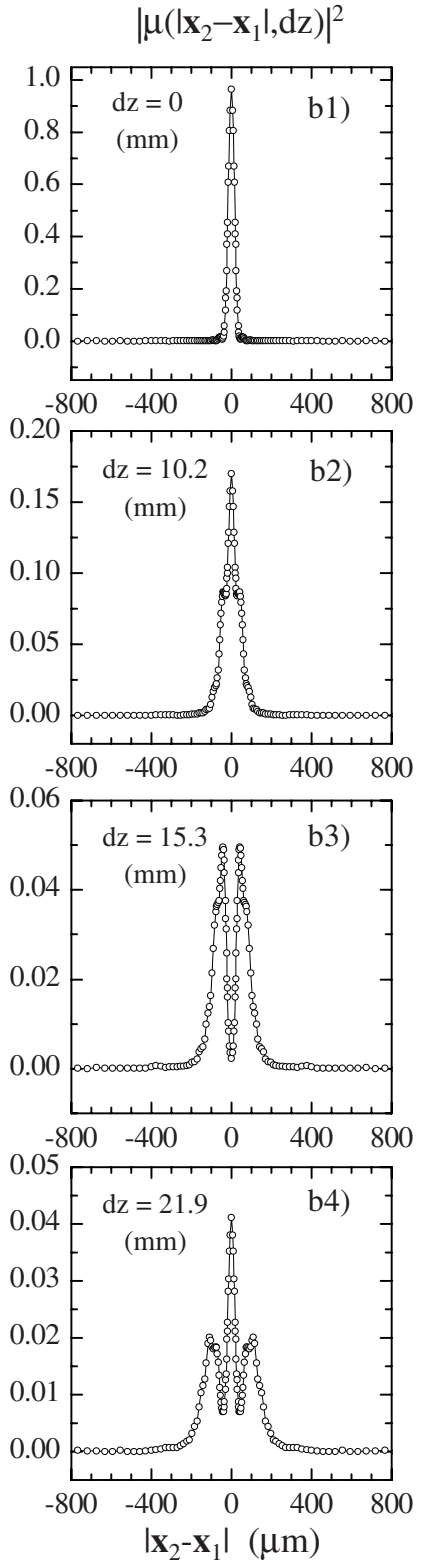
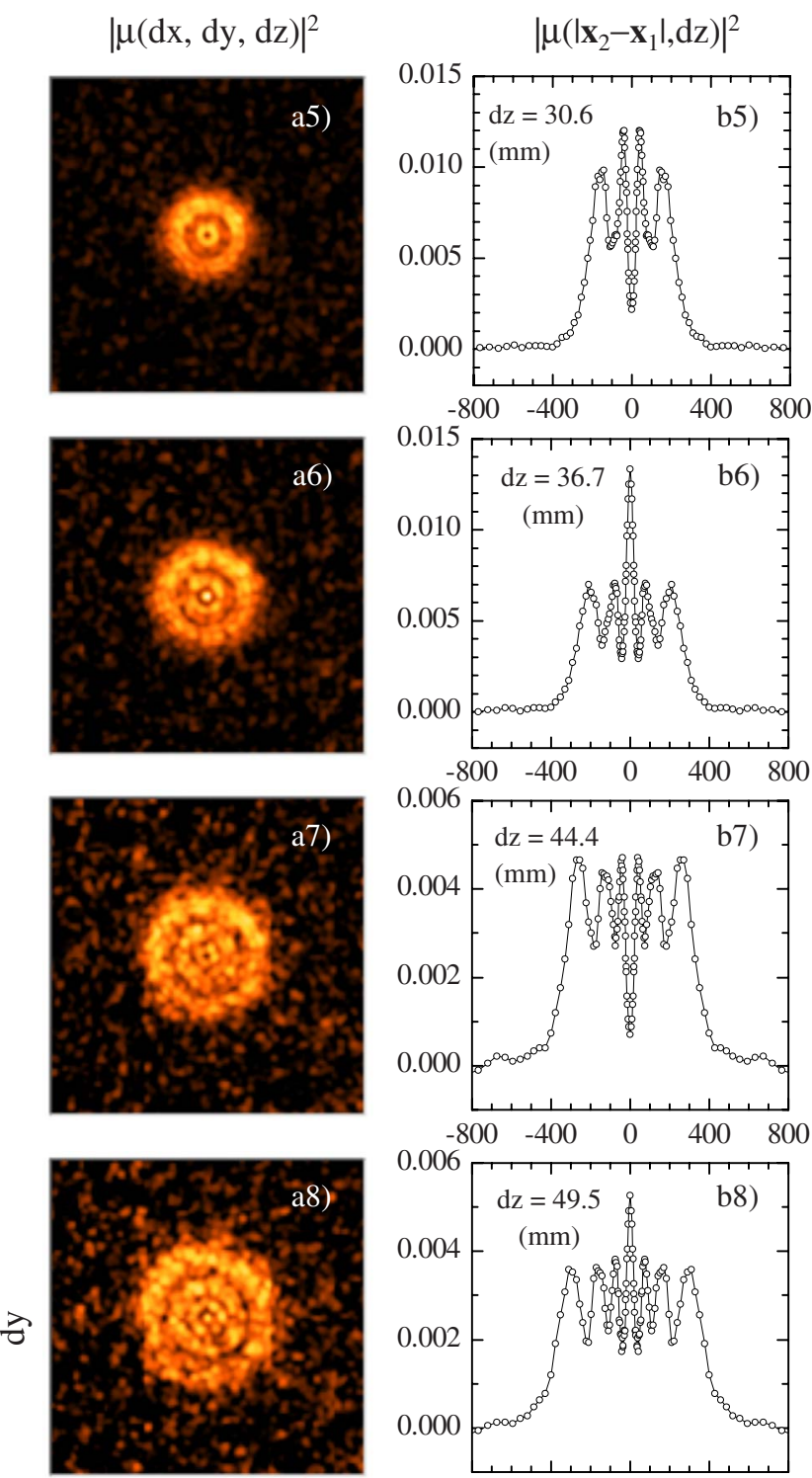

$\mathrm{dx}$

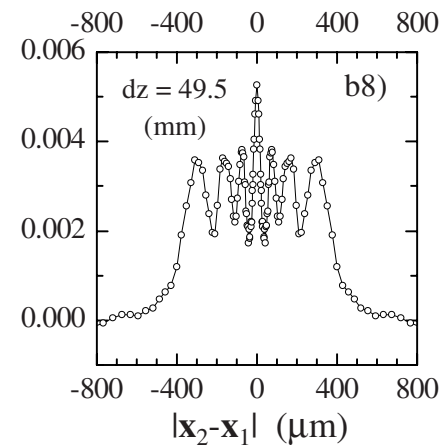

FIG. 14. (Color online) Experimental characterization of the 3D-coherence properties of Airy speckles in the deep-Fresnel zone showing the coherence factor $\left|\mu\left(\mathbf{x}_{2}-\mathbf{x}_{1}, d z\right)\right|^{2}$ at eight different distances $d z=z_{2}-z_{1}$. Panels (a1)-(a8) show the 2D transversal coherence factor as a function of $d x=x_{2}-x_{1}$ and $d y=y_{2}-y_{1}$, while panels (b1)-(b8) report their correspondent radial profiles as a function of $\left|\mathbf{x}_{2}-\mathbf{x}_{1}\right|$. The distances $d z$, indicated on panel (b1), have been chosen in correspondence of the maxima and minima exhibited by the $y-z$ intensity distribution indicated in panel (b2) of Fig. 13 with white vertical segments. Lines in panels (b) are guides for the eyes.

intensity profile illuminating its aperture is constant. In this way, the speckles formed in the focal plane of the lens $(z$ $=0$ plane) are characterized by a coherence factor given by the Fourier transform of the circular aperture [3], i.e.,

$$
\mathrm{C}_{0}\left(\mathbf{x}_{2}-\mathbf{x}_{1}\right)=\frac{2 J_{1}\left[\frac{\pi d\left|\mathbf{x}_{2}-\mathbf{x}_{1}\right|}{\lambda F}\right]}{\frac{\pi d\left|\mathbf{x}_{2}-\mathbf{x}_{1}\right|}{\lambda F}},
$$

where $J_{1}$ is the first-order Bessel function. The size of these speckles can be estimated by determining the full width half maximum (FWHM) of $\left|C_{0}\right|^{2}$. For the Airy speckles of our experiment, this size is

$$
\delta x_{0}^{\mathrm{FWHM}}=1.03 \frac{\lambda F}{d} \sim 34.7 \mu \mathrm{m},
$$

a figure which compares fairly well with our measured value of $\sim 35.6 \mu \mathrm{m}^{3}$

\footnotetext{
${ }^{3}$ Notice that these speckles are about $60 \%$ smaller than the Gaussian speckles of Sec. IV, whose FWHM width was equal to $\delta x_{0}^{\mathrm{FWHM}}=1.17 \delta x_{0} \sim 57.3 \mu \mathrm{m}\left(\delta x_{0} \sim 49 \mu \mathrm{m}\right)$.
} 

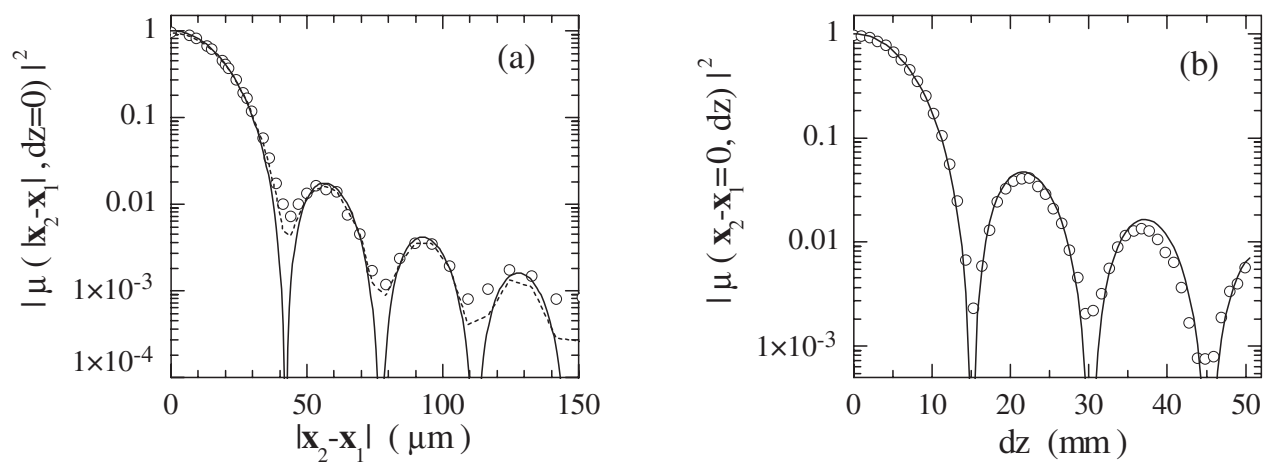

FIG. 15. Behavior of the 3D-coherence factor for Airy speckles in the deep-Fresnel zone: (a) Radial profile at $d z=0$ (circles) compared against theory [Eq. (23)] without (solid curve) and by taking into account (dashed curve) the finite size of the rings during the angular averaging procedure (see text). The speckle size is $\delta_{\mathrm{FWHM}}^{x_{0}} \sim 36 \mu \mathrm{m}$. (b) Longitudinal profile taken at the peak value $\left(\mathbf{x}_{2}=\mathbf{x}_{1}\right)$ as a function of $d z$ (circles) and comparison with theory [solid curve, Eq. (26)]. The longitudinal speckle size, defined as the distance at which $|\mu|^{2}$ $=0.5$, is $\delta z_{n} \sim 6.7 \mathrm{~mm}$.

The 3D morphology of Airy speckles is remarkably different from that of Gaussian speckles and exhibits a much more structured shape. These features are shown in Fig. 13, where the transversal $\left|\mu\left(\mathbf{x}_{2}-\mathbf{x}_{1}, d z=0\right)\right|^{2}$ [panels (a1) and (a2)] and longitudinal $\left|\mu\left(\mathbf{x}_{2}-\mathbf{x}_{1}, d z\right)\right|^{2}$ [panels (b1) and (b2)] behaviors of the coherence factor are reported at two different intensity-scale levels. Panels (a1) and (b1) are shown on a standard linear intensity scale, while panels (a2) and (b2) are highly saturated for enhancing visibility of all the details (oscillations, rings, dips, etc.) featuring the structure of the coherence factor. In particular one can appreciate that in panel (b2), in correspondence of the coherence optical axis $\left(\mathbf{x}_{2}=\mathbf{x}_{1}\right)$, there are many minima and maxima which keep alternating well beyond the distance at which $|\mu|^{2}$ has faded out $[\sim 5-10 \mathrm{~mm}$; see panel (b1)]. The longitudinal positions of these maxima and minima are indicated by the eight vertical white segments reported on the bottom axis of panel (b2). Their corresponding transversal coherence factors $\left|\mu\left(\mathbf{x}_{2}-\mathbf{x}_{1}, d z\right)\right|^{2}$ are reported in Fig. 14. For each of the eight distances $d z$, this figure shows the $2 \mathrm{D}$ coherence factor as a function of $d x=x_{2}-x_{1}$ and $d y=y_{2}-y_{1}$ [panels (a)] and its correspondent angularly average profile as a function of the radial distance $\left|\mathbf{x}_{2}-\mathbf{x}_{1}\right|$ [panels (b)]. One can appreciate that, in spite of the fact that $|\mu|^{2}$ becomes very small with increasing $d z$ [ $\sim 0.2 \%$ in panel (b8)], the radial profiles [panel (b)] of $|\mu|^{2}$ exhibit neat coherence rings, which resemble the "Fresnel diffraction" pattern of a round aperture. We will comment about this similarity at the end of this section. A quantitative analysis of the features described in Figs. 13 and 14 can be appreciated in Fig. 15, where we have reported the transversal (angularly averaged) profile of $\mid \mu\left(\mathbf{x}_{2}-\mathbf{x}_{1}, d z\right.$ $=0)\left.\right|^{2}$ as a function of $\left|\mathbf{x}_{2}-\mathbf{x}_{1}\right|[$ panel (a)] and the longitudinal profile $\left|\mu\left(\mathbf{x}_{2}-\mathbf{x}_{1}=0, d z\right)\right|^{2}$ taken in correspondence of its maximum $\left(\mathbf{x}_{2}=\mathbf{x}_{1}\right)$ [panel (b)]. Figure 15 shows that the transversal coherence profile in $d z=0$ (open symbols) is accurately matched by the Airy function, that is, by the squared modulus of Eq. (31) (solid curve). The discrepancies between theory and data in correspondence of the minima are due to unavoidable smoothing effects associated to ring thickness during the angular averaging procedure. The dashed curve shows the same effect on the theoretical curve and demonstrates that data and theory match quite well even at coherence levels of $0.1 \%$.

The longitudinal coherence on axis $\left(\mathbf{x}_{2}=\mathbf{x}_{1}\right)$ is reported in Fig. 15(b) (circles) and as expected on the basis of Fig. 14 exhibits several oscillations. The theoretical behavior describing these data can be found by using the general expression for the 3D-coherence factor in the deep-Fresnel zone (Eq. 16b of Ref. [1])

$$
\mu\left(\mathbf{x}_{2}-\mathbf{x}_{1}, d z\right)=\int C_{0}\left(\mathbf{x}^{\prime}\right) e^{i\left|\mathbf{x}_{2}-\mathbf{x}_{1}-\mathbf{x}^{\prime}\right|^{2} k /(2 d z)} \frac{d^{2} \mathbf{x}^{\prime}}{i \lambda d z}
$$

and substituting in it the function $C_{0}(\mathbf{x})$ given by Eq. (23). This calculation can be easily performed on axis $\left(\mathbf{x}_{2}=\mathbf{x}_{1}\right)$ and gives

$$
|\mu(0, d z)|^{2}=\operatorname{sinc}^{2}\left[\frac{\pi d z}{8 \lambda}(d / F)^{2}\right],
$$

where $\operatorname{sinc}(x)=\sin (x) / x$. The behavior predicted by Eq. (26) is reported in Fig. 15(b) as a solid curve and matches quite accurately the data even at amplitudes of the order of $0.1 \%$. With the help of Eq. (26), we can define the longitudinal length of the speckles as the value of $d z$ at which $|\mu(0, d z)|^{2}=0.5$, that is,

$$
z_{d}=3.55 \frac{\lambda F^{2}}{d^{2}} \sim 6.38 \mathrm{~mm}
$$

a figure which compares fairly well with a measured value of $6.7 \mathrm{~mm}$.

It is worth pointing out that Eq. (26) coincides with the on-axis intensity profile of a large collimated beam focused by a lens of diameter $d$ and focal length $F$ at a distance $d z$ from the focal point [14]. This result is consistent with Eq. (25) because "in the deep-Fresnel region the coherence function between two planes separated by a distance $d z$ evolves with $d z$ by following the free propagation of a light beam having $C_{0}(\mathbf{x})$ as its field distribution at $d z=0$ " (see [1]). It is also worth mentioning that Eq. (26) is equal (with $z=F$ ) to Eqs. (4)-(77) of Ref. [4], which, different from Eq. (26), was derived for the VCZ zone. This equivalence is not surprising because the equation in Ref. [4] was derived under the as- 
sumption $d z / z_{1} \ll 1$, which is equivalent to $z_{1} \ll z_{\mathrm{Fr}}$. Thus, as expected, the longitudinal coherence in the $\mathrm{VCZ}$ region far from the Fraunhofer zone follows the same pure diffractive behavior observed in the deep-Fresnel zone.

Finally we can comment about the similarity between the coherence rings reported in Fig. 14 and the rings observed in the Fresnel diffraction pattern of a round aperture. Again, this equivalence is exactly what predicted by Eq. (25): since the coherence factor in Eq. (23) is the Fourier transform of a round aperture, its propagated version will approach asymptotically $(d z \rightarrow \infty)$, the shape of a round aperture. But this behavior will take place only at $d z=\infty$, and for all the intermediate finite $d z$ 's, the neat Fresnel rings of a round aperture will appear, with the number of rings increasing with $d z$.

\section{CONCLUSIONS, POTENTIAL APPLICATIONS AND PERSPECTIVES}

In this work we have provided a detailed experimental characterization of the 3D-coherence properties of the light speckles produced by a pseudothermal source and we have studied the behavior of the coherence factor $\mu$ as a function of the distance from the source. Our findings match quite accurately the theoretical predictions reported in our recent companion paper [1], according to which the longitudinal coherence properties of the speckles change drastically with the distance from the source, with the speckle length being governed by a pure diffracting behavior $\left(\delta z \sim \delta x^{2} / \lambda\right)$ in the deep-Fresnel zone and becoming infinite in the Fraunhofer zone.

In order to investigate such a long range of distances, we had to use different tunable pseudothermal sources capable of producing speckles with the desired characteristics. The requirement of having a tunable thermal source was of great importance because, while VCZ speckles are easily observable at any sufficiently large distance $\left(z>z_{\mathrm{VCZ}}\right)$ and are controlled only by the profile and size $D$ of the beam impinging onto a physical diffuser (such as a ground glass or a scattering sample), for deep-Fresnel speckles the situation is definitely more difficult. Deep-Fresnel speckles depend on the microscopic details of the source and their properties (transversal size and longitudinal length) are set by the shape and size $\delta x_{0}$ of the source grains, which, in the case of a physical diffuser, are fixed features of the particular glass or scattering particles being used. Typically, $\delta x_{0}$ is of the order of microns, so that the detection of such small and short speckles, although possible $[15,16]$, would be rather troublesome, with the necessity of using high magnification optics. Furthermore, with such small speckles, the deep-Fresnel zone would be fairly short and distances $z<z_{\mathrm{VCZ}}$ would be hardly accessible even with a simple setup as the one described in Fig. 1.

To overcome these difficulties we realized synthetic, tunable, planar sources capable of producing speckles with the desired shape (either Gaussian or Airy) and tunable size of the order of several tens of microns. The realization of this kind of highly flexible Fourier transform thermal sources is one of the by-products of our work. Furthermore, we believe that the possibility of controlling the overall wave front curvature of the speckles and realizing in such a way a nonplanar thermal source could be very useful, for example, for producing VCZ speckles at close distance or for stretching the zone where deep-Fresnel speckles are observed.

As known, the 3D-coherence properties of light speckles are of great interest in many classical applications of speckle metrology, such as speckle photography, interferometry, and holography [4]. More recently, it turned out to be a relevant issue in novel optical imaging techniques, such as dynamic speckle illumination microscopy $[17,18]$ and ghost imaging $[19,20]$. In both cases, the sample is illuminated with a time varying speckle pattern whose transversal and longitudinal coherence sizes establish the transversal and longitudinal resolutions of the image formation. Thus, if the speckles are very small and short it is possible to realize a high resolution 3D imaging of the sample either by adopting a confocal-like optical setup as proposed by Mertz and co-workers $[17,18]$ or by exploiting the potentiality of the ghost imaging technique [20]. Conversely, very long speckles could be used in imaging systems where it is important to detect the presence of objects inside a $3 \mathrm{D}$ volume with a good transversal but no longitudinal resolution, as it could be of interest for particle sizing or remote sensing applications.

Another by-product of our work is the possibility of measuring the 3D correlation of the point spread function (PSF) of an optical wide angle microscope by using a setup similar to the one described in Fig. 12. Indeed, if the diameter $d$ of the aperture is made equal to the lens diameter, each speckle carries the imprinting of the lens PSF, and the coherence factor $|\mu|^{2}$ would provide quantitative information on the 3D (intensity) PSF of the lens. A qualitative demonstration of this statement can be done by comparing Figs. 13 and 14 of our work with standard PSF images, as for example, the ones reported in Ref. [21].

Finally, we would like to point out that, while all the data reported in this work are relative to the squared modulus coherence factor $|\mu|^{2}$, it would be quite straightforward to measure also the (complex) coherence factor $\mu$, with the same, slightly modified, setup of Fig. 1. Indeed, one could make the two speckle beams overlap at an angle and produce onto the detector plane an interferogram of straight fringes. In that case, the recovery of the fringes contrast and position would provide a direct measure of the amplitude and phase of $\mu$, respectively.

\section{ACKNOWLEDGMENTS}

We are grateful to B. E. A. Saleh, M. Giglio, M. A. C. Potenza, M. D. Alaimo, and R. Cerbino for useful discussions. D.M. and F.F. acknowledge the work of M. D. Alaimo on the longitudinal coherence properties of speckle light in the deep-Fresnel region [22]. 
[1] A. Gatti, D. Magatti, and F. Ferri, Phys. Rev. A 78, 063806 (2008).

[2] Laser Speckle and Related Phenomena, Topics in Applied Physics Vol. 9, edited by D. Dainty (Springer, Berlin, 1975).

[3] J. W. Goodman, Statistical Optics (Wiley-Interscience, New York, 1985).

[4] J. W. Goodman, Speckle Phenomena in Optics (Roberts, Greenwood, CO, 2007).

[5] L. Leushacke and M. Kirchner, J. Opt. Soc. Am. A 7, 827 (1990).

[6] Q. B. Li and F. P. Chiang, Appl. Opt. 31, 6287 (1992).

[7] J. W. Goodman, Speckle Phenomena in Optics (Roberts, Greenwood, CO, 2007), Chap. 4, pp. 82-84.

[8] M. Giglio, M. Carpineti, and A. Vailati, Phys. Rev. Lett. 85, 1416 (2000).

[9] F. Ferri, D. Magatti, D. Pescini, M. A. C. Potenza, and M. Giglio, Phys. Rev. E 70, 041405 (2004).

[10] R. Cerbino, Phys. Rev. A 75, 053815 (2007).

[11] P. H. Van Cittert, Physica (Utrecht) 1, 202 (1934); F. Zernike, ibid. 5, 785 (1938).

[12] J. W. Goodman, Statistical Optics (Springer, Berlin, 1975),
Chap. 5, pp. 218-222.

[13] M. Born and E. Wolf, Principles of Optics (Pergamon, New York, 1908).

[14] M. Born and E. Wolf, Principles of Optics (Pergamon, New York, 1908), Chap. 8.8, pp. 435-449.

[15] M. Giglio, M. Carpineti, A. Vailati, and D. Brogioli, Appl. Opt. 40, 4036 (2001).

[16] M. Bache, D. Magatti, F. Ferri, A. Gatti, E. Brambilla, and L. A. Lugiato, Phys. Rev. A 73, 053802 (2006).

[17] C. Ventalon and J. Mertz, Opt. Lett. 30, 3350 (2005).

[18] C. Ventalon, R. Heintzmann, and J. Mertz, Opt. Lett. 32, 1417 (2007).

[19] F. Ferri, D. Magatti, A. Gatti, M. Bache, E. Brambilla, and L. A. Lugiato, Phys. Rev. Lett. 94, 183602 (2005).

[20] F. Ferri, D. Magatti, and A. Gatti, Appl. Phys. Lett. 92, 261109 (2008).

[21] M. B. Cannell, A. Mc Morland, and C. Soeller, Handbook of Biological Confocal Microscopy, editor by J. B. Pawley (Springer, New York, 2006), Chap. 25.

[22] M. D. Alaimo, Ph.D. thesis, University of Milan, Italy, 2006. 